\title{
Nuevo Liolaemus (Iguania: Liolaemidae) con novedoso carácter morfológico, de la frontera entre Argentina y Chile
}

\author{
Cristian Simón Abdala, Marcos Maximiliano Paz \& Romina Valeria Semhan \\ CONICET - Instituto de Herpetología, Fundación Miguel Lillo, Miguel Lillo 251, Tucumán 4000, Argentina; \\ samiryjazmin@gmail.com,marcosmax_paz@hotmail.com,romisemhan@gmail.com
}

\author{
Recibido 10-VIII-2012. Corregido 05-IV-2013. Aceptado 06-V-2013.
}

\begin{abstract}
New Liolaemus (Iguania: Liolaemidae) with a new morphological character from the border between Argentina and Chile. Liolaemus belongs to the Eulaemus subgenus, that is included in the Liolaemus montanus group or section, and is distributed in Argentina, Bolivia, Chile and Peru. The species members of this clade inhabit arid and high altitude environments, usually above $2800 \mathrm{~m}$, and have a strong tendency to herbivory and a viviparous reproductive type. In a herpetological expedition to the Salta Province highlands, Argentina, in April 2012, we collected individuals of this new species for their description. The specimens were collected with loop, slaughtered by anesthesia and deposited in the herpetological collection of Fundación Miguel Lillo in Tucumán (FML). To characterize this new taxon, we analyzed 144 external morphological characters, referring primarily to lepidosis, color pattern, and body proportions, comparing with data from 66 constituent species of the L. montanus group (45 taxa from collection specimens and 21 from literature). Description of the colors in life was made in the field and based on photographs taken during the capture. The species described in this work inhabits a very narrow mountain range area in Salta Province (Argentina), and the Atacama Region (Chile), always above the $3600 \mathrm{~m}$. This new taxon shows characteristics of lepidosis and color patterns clearly different from the other species members of the L. montanus group: the maximum snout-vent length is $72.9 \mathrm{~mm}$; shows 74-96 scales around midbody, 89-104 dorsal scales between the occiput and hind limbs, 92-109 ventrals, precloacal pores are evident in both, males and females, and supernumerary pores in males. Also, the presence of postcloacal pores stands out as unique among all Liolaemus. This is a new character, not only for the genus, but also to all Reptilia, becoming a morphological discovery of great importance, since in reptiles only precloacal and femoral pores are known. Based on our field observations, this new Liolaemus is related to rocky environments, which are used as refuge by this omnivorous species, and when handled, very aggressive males. Rev. Biol. Trop. 61 (4): 1563-1584. Epub 2013 December 01.
\end{abstract}

Key words: Liolaemus, lizard, new species, postcloacales pores, cordillera, Reptilia.

La familia Liolaemidae está integrada por el género monoespecífico Ctenoblepharis, endémico de las costas de Perú, por Phymaturus, natural de Argentina y Chile, y por Liolaemus, el segundo género más diverso dentro de Iguania, con más de 245 especies (Avila et al. 2010, Lobo et al. 2010a, Abdala et al. 2012a). Liolaemus se encuentra distribuido desde el centro de Perú hasta Tierra del Fuego, en el extremo sur del continente americano, presentando las especies de lagartijas más australes del mundo (Abdala 2007). Dentro del género se puede encontrar una variedad muy interesante de tipos morfológicos (Cei 1993, Abdala 2007), así como de hábitos en la dieta (Martori et al. 1999, Espinoza et. al. 2004), reproducción (Ramírez-Pinilla 1991, Espinoza et. al. 2004, Martori \& Aun 2010) y comportamiento (Labra et al. 2007). Los aspectos filogenéticos de Liolaemus han sido también materia de estudio en los últimos años (Lobo 2005, Abdala 2007, Abdala et al. 2012b), al igual que los biogeográficos (Díaz Gómez 2007a, 2007b). Sin embargo, la taxonomía es la rama de la biología que ha sido desarrollada en todo su potencial dentro del género, 
describiéndose 69 nuevas especies solo en los últimos diez años, entre las que se destacan aquellas dadas a conocer en los trabajos de Abdala (2005), Abdala et al. (2008), Abdala \& Quinteros (2008), Avila et al. (2011), Breitman et al. (2011).

Con base en las diferentes propuestas sistemáticas presentadas, en este trabajo se describe un nuevo lagarto del género Liolaemus hallado en la cordillera de los Andes de la provincia de Salta, desde el límite entre Argentina y Chile hasta aproximadamente $29 \mathrm{~km}$ al Este, siguiendo la ruta provincial $160 \mathrm{y}$ por encima de los 3 600msnm. Este nuevo Liolaemus pertenecería al subgénero Eulaemus y dentro de este subgénero, sería integrante del grupo de Liolaemus montanus no boulengeri. El grupo de L. montanus es muy diverso, estaría integrado por más de 60 especies (Lobo et al. 2010a, Quinteros \& Abdala 2011) que habitan a elevada altitud, generalmente por encima de los $2800 \mathrm{msnm}$, aparentemente serían especies vivíparas y siempre relacionadas a climas andinos (Ramírez-Leyton \& Pincheira-Donoso 2005, Valladares 2004). En los últimos años se describieron 15 nuevas especies del grupo Liolaemus montanus (Abdala et al. 2008, Lobo et al. 2010b, Quinteros \& Abdala 2011), principalmente relacionadas o emparentadas con $L$. dorbignyi Koslowsky 1898; L. multicolor Koslowsky 1898 y L. andinus Koslowsky 1895. Sin embargo, estas hipótesis de parentesco se basan sólo en presunciones taxonómicas, ya que no se ha realizado una filogenia formal que agrupe a las especies del grupo de L. montanus.

En este trabajo, se presenta y describe formalmente una nueva especie de Liolaemus, y se reporta la presencia de un carácter morfológico externo muy particular y novedoso. Dicha característica anatómica, es un atributo de esta nueva especie de lagartija y no ha sido registrado en ninguna otra especie de reptil. Asimismo, en esta investigación se compara detalladamente el nuevo taxón con especies afines, y se discuten sus posibles relaciones de parentesco dentro del grupo de L. montanus.

\section{MATERIALES Y MÉTODOS}

Los ejemplares fueron recolectados en abril del 2012, con ayuda de un lazo, y fueron sacrificados inmediatamente por sobre-anestesia, fijados en formol al $10 \%$ y conservados en alcohol al 70\%. Se examinaron ejemplares de 45 de las 66 especies pertenecientes al grupo de L. montanus que se listan en el Apéndice I. Se utilizaron 144 caracteres morfológicos externos, referidos principalmente a lepidosis o escamación, patrón de coloración y proporciones corporales, corrientemente descritos o citados principalmente por Laurent (1985), Lobo (2001) y Abdala (2007). La descripción de los colores en vida fue realizada en el campo y en base a fotografías tomadas durante la captura de los individuos. Las observaciones de lepidosis y medidas corporales se obtuvieron mediante lupa binocular y un calibre o Vernier digital Mitutoyo con precisión de $0.01 \mathrm{~mm}$. La terminología referida a los pliegues del cuello sigue a Frost (1992) y a Abdala (2007), la del patrón de coloración del cuerpo sigue a Lobo \& Espinoza (1999) y a Abdala (2007). De las especies que no se pudo adquirir ni observar material, o de las cuales se analizaron pocos ejemplares, se obtuvo información de la literatura (Navarro \& Núñez 1993, Núñez \& Yánez 1983, Laurent 1992, 1998, Valladares et al. 2002, Núñez et al. 2003, Valladares 2004, 2011, Ibarra-Vidal 2005, Pincheira-Donoso \& Núñez 2005, Ramírez-Leyton \& PincheiraDonoso 2005, Pincheira-Donoso et al. 2008, Langstroth 2011).

Todos los lagartos utilizados en este estudio están depositados en la colección Herpetológica de la Fundación Miguel Lillo de Tucumán (FML), y en la colección Herpetológica del Museo de Ciencias Naturales de Salta (MCN).

\section{RESULTADOS}

Liolaemus porosus sp. nov. (Cuadros 1 y 2; Figs. 1-5)

Holotipo (Fig. 1): FML 2497. Macho adulto. A $20 \mathrm{~km}$ al Este del puesto de Gendarmería 


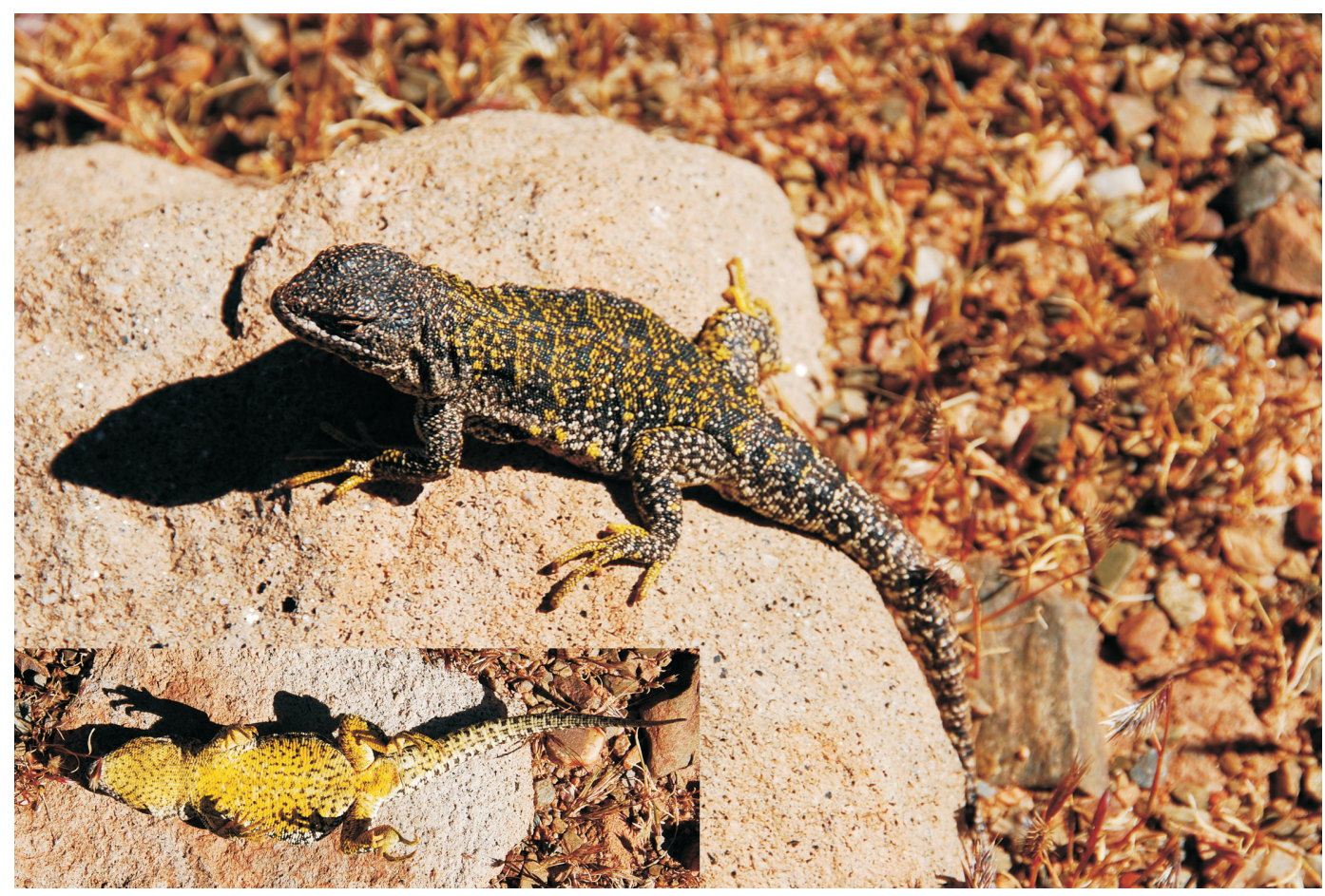

Fig. 1. Vista dorsal y ventral del holotipo de Liolaemus porosus sp. nov.

Fig. 1. Dorsal and ventral views of Liolaemus porosus sp. nov. holotype.

Argentina, ubicado en el límite internacional con Chile por el paso Socompa, departamento de Los Andes, provincia de Salta, Argentina. Coordenadas: $24^{\circ} 34^{\prime} 40.2^{\prime \prime}$ S y $68^{\circ} 11^{\prime} 58.4^{\prime \prime} \mathrm{W}$, $3707 \mathrm{msnm}$.

Paratipos: FML 24772. Hembra. A $23 \mathrm{~km}$ al Este del puesto de Gendarmería Argentina, ubicado en el límite internacional con Chile por el paso Socompa, departamento de Los Andes, provincia de Salta, Argentina. Coordenadas: $24^{\circ} 36^{\prime} 16.5^{\prime \prime} \mathrm{S}$ y $68^{\circ} 12^{\prime} 33.1^{\prime \prime} \mathrm{W}, 3861 \mathrm{msnm}$.

FML 24773. Macho. Igual datos que el Holotipo.

FML 24774. Macho. A $13 \mathrm{~km}$ al Noreste de la estación ferroviaria de Chuculaqui, camino al paso Socompa, departamento de Los Andes, provincia de Salta, Argentina. Coordenadas: $24^{\circ} 40^{\prime} 17.3^{\prime}$ S y $68^{\circ} 09^{\prime} 18.5^{\prime \prime} \mathrm{W}, 4209 \mathrm{msnm}$.

FML 24778-79. Dos ejemplares hembras. A $29 \mathrm{~km}$ al Este del puesto de Gendarmería Argentina, ubicado en el límite internacional con Chile por el paso Socompa, departamento de Los Andes, provincia de Salta, Argentina. Coordenadas: $24^{\circ} 38^{\prime} 39.8^{\prime \prime}$ S y $68^{\circ} 11^{\prime} 22.6^{\prime \prime} \mathrm{W}$.

FML 24780. Macho. Ladera Este del Volcán Socompa, sobre la Ruta provincial 163, departamento de Los Andes, provincia de Salta, Argentina. Coordenadas: $24^{\circ} 28^{\prime} 24.1^{\prime \prime} \mathrm{S}$ y $68^{\circ} 14$ ' 44.0 "' W.

Diagnosis: Dentro de la serie de Liolaemus montanus, L. porosus es la única especie que presenta machos con poros postcloacales (1-8 $x=3.0$ en cinco de los seis machos). También se diferencia de L. annectens Boulenger 1901; L. chlorostictus Laurent 1993; L. dorbignyi, L. duellmani Cei 1978; L. fabiani Núñez \& Yañez 1983; L. filiorum Ramírez-Leyton \& Pincheira-Donoso 2005; L. forsteri Laurent 1982; L. foxi Núñez 2000; L. huayra Abdala et al. 2008; L. inti Abdala et al. 2008; L. jamesi (Boulenger 1891); L. melanogaster Laurent 
1998; L. nigriceps Philippi 1960; L. orientalis Müller 1924; L. patriciaturrae Navarro 1993; L. pachecoi Laurent 1995; L. pleopholis Laurent 1998; L. polystictus Laurent 1992; L. puritamensis Núñez \& Fox 1989; L. robustus Laurent 1992; L. scrocchii Quinteros et al. 2008; L. thomasi Laurent 1998; L. vallecurensis Pereyra 1992; L. vulcanus Quinteros \& Abdala 2011; y L. williamsi Laurent 1992, porque todas estas especies son de mayor tamaño (tienen un máximo largo hocico-cloaca en los adultos, entre 77 y $105 \mathrm{~mm}$ vs. $72.9 \mathrm{~mm}$ en $L$. porosus). Se diferencia de L. audituvelatus Núñez \& Yáñez 1983; L. erguetae Laurent 1995; L. erroneus Núñez \& Yáñez 1983; L. etheridgei Laurent 1998; L. famatinae Cei 1980; L. fittkaui Laurent 1986; L. insolitus Cei 1982; L. lopezi Ibarra-Vidal 2005; L. manueli Núñez et al. 2003; L. ortizi Laurent 1982; L. pantherinus Pelligrin 1909; L. poconchilensis Valladares 2004; L. reichei Werner 1907; L. rosenmanni Núñez 1992; L. ruibali DonosoBarros 1961; L. schmidti Marx 1960; L. stolzmanni Steindachner 1891 y L. torresi Núñez 2003, por ser especies de menor tamaño que L. porosus, (LHCmax 48-66mm en adultos vs. $72.9 \mathrm{~mm}$ en L. porosus).

L. porosus se caracteriza por tener escamas dorsales del cuerpo yuxtapuestas o subyuxtapuestas, laminares, sin quilla; caracteres que lo diferencian de Liolaemus disjunctus Laurent 1990; L. etheridgei, L. fittkaui, L. huacahuasicus Laurent 1985; L. montanus Koslowsky 1898; L. orko Abdala \& Quinteros 2008; L. ortizi, L. polystictus, L. thomasi y L. tropidonotus Boulenger 1901 que presentan escamas del dorso del cuerpo imbricadas con quillas evidentes. La disposición y ausencia de quilla en las escamas dorsales también diferencian a L. porosus de L. dorbignyi, L. famatinae, L. griseus Laurent 1984; L. inti, L. huayra, L. jamesi, L. melanogaster, L. pleopholis, L. pulcherrimus Laurent 1991; L. signifer Duméril \& Bibron 1837; y L. williamsi que tienen escamas imbricadas o subimbricadas con quilla leve.

El número de escamas alrededor del cuerpo en L. porosus varía entre 74 y $96(\mathrm{x}=88.0)$, carácter que lo diferencia de varias especies del grupo que tienen hasta 73 escamas, como en $L$. annectens, L. audituvelatus, L. chlorostictus, $L$. disjunctus, $L$. dorbignyi, $L$. etheridgei, $L$. fabiani, L. famatinae, L. fittkaui, L. griseus, L. huacahuasicus, L. huayra, L. insolitus, L. islugensis Ortiz \& Marquet 1987; L. jamesi, L. lopezi, L. melanogaster, L. montanus, L. orientalis, L. orko, L. ortizi, L. pachecoi, L. poconchilensis, L. polystictus, L. puritamensis, L. robustus, L. scrocchii, L. stozlmanni, L. thomasi, L. torresi, L. tropidonotus, L. vulcanus y L. williamsi.

El número de escamas dorsales entre el occipucio y los miembros posteriores en Liolaemus porosus varía entre 89 y 104 ( $\mathrm{x}=95.6)$, y es mayor que en las siguientes especies que tienen hasta 88 escamas: $L$. annectens, $L$. audituvelatus, $L$. chlorostictus, $L$. dorbignyi, $L$. etheridgei, L. fittkaui, L. griseus, L. huacahuasicus, L. huayra, L. inti, L. islugensis, L. jamesi, L. melanogaster, L. montanus, L. orientalis, $L$. orko, L. pachecoi, L. poconchiliensis, L. polystictus, L. puritamensis, L. robustus, L. scrocchii, L. thomasi, L. vulcanus, y L. williamsi.

El número de escamas ventrales entre la mental y el borde de la cloaca en Liolaemus porosus varía entre 92 y 109 ( $\mathrm{x}=99.8)$, y es mayor que en las siguientes especies que tienen hasta 91 escamas ventrales: L. annectens, L. chlorostictus, L. etheridgei, L. fabiani, L. famatinae, L. fittkahui, L. forsteri, L. griseus, L. huacahuasicus, L. huayra, L. islugensis, $L$. jamesi, L. melanogaster, L. montanus, L. orientalis, L. pachecoi, L. polystictus, L. pulcherrimus, L. puritamensis, L. robustus, L. ruibali, L. signifer, L. thomasi, y L. williamsi.

En L. porosus el número de escamas en el cuello, desde el borde posterior del oído hasta el miembro anterior, siguiendo el pliegue longitudinal, varía entre 47 y 51 ( $\mathrm{x}=49.0)$, y es mayor que en las siguientes especies que tienen hasta 45 escamas en el cuello: $L$. annectens, $L$. audituvelatus, L. dorbignyi, L. eleodori Cei, Etheridge \& Videla 1985; L. fabiani, L. famatinae, L. fittkahui, L. forsteri, L. foxi, L. halonastes, L. huacahuasicus, L. islugensis, L. jamesi, L. melanogaster, L. montanus, L. orientalis, $L$. orko, L. pachecoi, L. puritamensis, L. robertoi 
Pincheira-Donoso \& Núñez 2004; L. robustus, L. ruibali, L. scrocchii, L. schmidti, L. signifer, L. thomasi, L. torresi, y L. vulcanus.

El número de escamas gulares en Liolaemus porosus varía entre 41 y $52(\mathrm{x}=46.1)$ y es mayor que en las siguientes especies que tienen hasta 40 escamas gulares: L. annectens, L. chlorostictus, L. erguetae, L. etheridgei, L. fabiani, L. famatinae, L. fittkaui, L. griseus, $L$. huacahuasicus, L. huayra, L. jamesi, L. melanogaster, L. montanus, $L$. orientalis, $L$ orko, $L$. pachecoi, L. pleopholis, L. polystictus, L. pulcherrimus, L. robertoi, L. robustus, L. signifer, L. thomasi, y L. torresi.

El número de poros precloacales en los machos de Liolaemus porosus varía entre cinco $\mathrm{y}$ siete $(\mathrm{x}=5.8)$ y es mayor que en las siguientes especies que tienen hasta cinco poros precloacales $(\mathrm{x}<4.8)$ : L. halonastes, L. manueli y $L$. poconchiliensis.

El número de poros precloacales en las hembras de Liolaemus porosus varía entre cuatro y cinco $(x=4.4)$, diferenciándose de las siguientes especies que carecen de poros: L. audituvalatus, L. filiorum. L. fittkaui, L. forsteri, L. hajeki Núñez et al. 2004; L. halonastes, L. jamesi, L. lopezi, L. melanogaster, L. molinai Valladares et al. 2002; L. ortizi, L. poconchiliensis, L. polystictus, L. puritamensis, L. robertoi, L. ruibali, L. torresi, y L. vallecurensis. También de las especies que tienen hembras con hasta cuatro poros precloacales $(\mathrm{x}<$ 3.0): L. andinus $(\mathrm{x}=2.0)$, L. annectens $(\mathrm{x}=0.5)$, L. eleodori $(\mathrm{x}=2.7)$, Liolaemus gracielae Abdala et al. $2009(\mathrm{x}=0.3) ;$ L. multicolor $(\mathrm{x}=0.1), L$. orientalis $(\mathrm{x}=1.8), \mathrm{y}$ L. poecilochromus Laurent $1986(\mathrm{x}=0.3)$.

El número de poros supernumerarios en los machos de Liolaemus porosus varía entre uno y $12(\mathrm{x}=5.5)$ diferenciándose de todas las especies del grupo de L. montanus que carecen de poros supernumerarios, excepto L. cazianae Lobo et al. 2010b; L. famatinae, L. forsteri, L. griseus, L. huayra, L. melanogaster, L. orientalis, L. orko, L. pulcherrimus, $L$. signifer y $L$. thomasi; sin embargo, también se diferencia de todas las especies mencionadas anteriormente porque éstas tienen una media muy inferior a la de L. porosus (max. L. griseus con $\mathrm{x}=2.3$ ).

English diagnosis: Within the Liolaemus montanus series, $L$. porosus is the only species which shows postcloacal pores in males (1-8 x $=3.0$ in five of the six males). It can be distinguished from L. annectens; L. chlorostictus; $L$. dorbignyi; L. duellmani; L. fabiani; L. filiorum; L. forsteri; L. foxi; L. huayra; L. inti; L. jamesi ; L. melanogaster; L. nigriceps; L. orientalis; L. patriciaturrae, L. pachecoi ; L. pleopholis; L. polystictus; L. puritamensis; L. robustus, L. scrocchii; L. thomasi; L. vallecurensis; $L$. vulcanus, and $L$. williamsi, because all these species are larger (with a maximum snoutvent length in adults, between 77 and $105 \mathrm{~mm}$ vs. $72.9 \mathrm{~mm}$ in $L$. porosus). It differs from $L$. audituvelatus; L. erguetae; L. erroneus; L. etheridgei; L. famatinae; L. fittkaui; L. insolitus; $L$. lopezi; L. manueli; L. ortizi; L. pantherinus; L. poconchilensis; L. reichei; L. rosenmanni; L. ruibali; L. schmidti; L. stolzmanni and $L$. torresi, because are smaller than $L$. porosus, (48-66mm maxSVL vs $72.9 \mathrm{~mm}$ in L. porosus).

$L$. porosus is characterized by juxtaposed or subyuxtaposed, laminar, and without keel dorsal body scales; character states that differentiate it from L. disjunctus; L. etheridgei, L. fittkaui, L. huacahuasicus; L. montanus; L. orko; L. ortizi, L. polystictus, L. thomasi and L. tropidonotus which exhibit dorsal scales imbricate with evident keel. Dorsal scales also differentiate $L$. porosus from $L$. dorbignyi, $L$. famatinae, L. griseus; L. inti, L. huayra, L. jamesi, L. melanogaster, L. pleopholis, L. pulcherrimus, L signifer and $L$. williamsi which have imbricated or subimbricated and slightly keeled dorsal scales.

The number of scales around the body in L. porosus varies between 74-96 ( $\mathrm{x}=88.0)$, differing from several species of the group which have up to 73 scales, as in L. annectens, L. audituvelatus, L. chlorostictus, L. disjunctus, L. dorbignyi, L. etheridgei, L. fabiani, L. famatinae, L. fittkaui, L. griseus, L. huacahuasicus, L. huayra, L. insolitus, L. islugensis; L. jamesi, L. lopezi, L. melanogaster, L. montanus, 
L. orientalis, L. orko, L. ortizi, L. pachecoi, L. poconchilensis, L. polystictus, L. puritamensis, L. robustus, L. scrocchii, L. stozlmanni, L. thomasi, L. torresi, L. tropidonotus, L. vulcanus, and L. williamsi.

The number of dorsal scales between occiput and hind limbs in L. porosus varies between 89 and $104(\mathrm{x}=95.6)$, and is higher than $L$. annectens, $L$. audituvelatus, $L$. chlorostictus, $L$. dorbignyi, L. etheridgei, L. fittkaui, L. griseus, L. huacahuasicus, L. huayra, L. inti, L. islugensis, L. jamesi, L. melanogaster, L. montanus, L. orientalis, L. orko, L. pachecoi, L. poconchiliensis, L. polystictus, L. puritamensis, L. robustus, L. scrocchii, L. thomasi, L. vulcanus, and $L$. williamsi, which this scales are not higher than 88 .

The number of ventral scales between mental and ventral edge of the cloaca in $L$. porosus is higher than in L. annectens, L. chlorostictus, L. etheridgei, L. fabiani, L. famatinae, L. fittkahui, L. forsteri, L. griseus, L. huacahuasicus, L. huayra, L. islugensis, L. jamesi, L. melanogaster, L. montanus, $L$. orientalis, L. pachecoi, L. polystictus, L. pulcherrimus, L. puritamensis, L. robustus, $L$. ruibali, L. signifer, L. thomasi, and $L$. williamsi the (92-109; $\mathrm{x}=99.8$ vs up to 91 , respectively).

In Liolaemus porosus the number of scales in neck (from posterior edge of auricular meatus to shoulder, along the longitudinal fold) varies between 47-51 ( $\mathrm{x}=49.0)$, being higher than $L$. annectens, $L$. audituvelatus, $L$. dorbignyi, L. eleodori; L. fabiani, L. famatinae, L. fittkahui, L. forsteri, L. foxi, L. halonastes, L. huacahuasicus, L. islugensis, L. jamesi, L. melanogaster, L. montanus, L. orientalis, $L$. orko, L. pachecoi, L. puritamensis, L. robertoi; L. robustus, L. ruibali, L. scrocchii, L. schmidti, L. signifer, L. thomasi, L. torresi, and L. vulcanus, which have up to 45 neck's scales.

The number of gular scales is higer in Liolaemus porosus than in L. annectens, $L$. chlorostictus, L. erguetae, L. etheridgei, L. fabiani, L. famatinae, L. fittkaui, L. griseus, $L$. huacahuasicus, L. huayra, L. jamesi, L. melanogaster, L. montanus, L. orientalis, L. orko, L. pachecoi, L. pleopholis, L. polystictus, L. pulcherrimus, L. robertoi, L. robustus, L. signifer, L. thomasi, and L. torresi $(41-52 ; \mathrm{x}=46.1$; vs up to 40 , respectively).

Number of precloacal pores in males in Liolaemus porosus $(5-7 ; \mathrm{x}=5.8)$ is higher than in the L. halonastes, L. manueli, and L. poconchiliensis $(\mathrm{x}=<4.8)$

The presence of precloacal pores in females $(4-5 ; \mathrm{x}=4.4)$ distinguish the new taxon from L. audituvalatus, L. filiorum, L. fittkaui, L. forsteri, L. hajeki, L. halonastes, L. jamesi, L. lopezi, L. melanogaster, L. molinai; L. ortizi, L. poconchiliensis, L. polystictus, L. puritamensis, L. robertoi, L. ruibali, L. torresi, and L. vallecurensis which lack precloacal pores. Also, differ from females with up to four (x $<3.0)$ precloacal pores: L. andinus $(\mathrm{x}=2.0)$, L. annectens $(\mathrm{x}=0.5)$, L. eleodori $(\mathrm{x}=2.7)$, Liolaemus gracielae $(\mathrm{x}=0.3)$, L. multicolor $(\mathrm{x}$ $=0.1)$, L. orientalis $(\mathrm{x}=1.8)$, and L. poecilochromus $(\mathrm{x}=0.3)$.

The number of supernumerary pores of males of Liolaemus porosus $(1-12 ; \mathrm{x}=5.5)$ differ from all species of the L. montanus group which lack supernumerary pores, with the exception of L. cazianae; L. famatinae, $L$. forsteri, L. griseus, L. huayra, L. melanogaster, L. orientalis, L. orko, L. pulcherrimus, $L$. signifer and L. thomasi, which exhibit supernumerary pores, but they have a lower number of pores (maximum datum registered from L. griseus $\mathrm{x}=2.3$ ).

Descripción (Cuadros 1 y 2 y Figs. 2-3): La nueva especie que se describe en este trabajo es referida al subgénero Eulaemus por tener el epimiso del pterigomandibularis pigmentado y la cabeza media del músculo flexor tibialis interno cubierto por el músculo puboisquiotibilis hipertrofiado. Dentro de este subgénero se asigna a la Sección de Liolaemus montanus (Schulte et al. 2000) porque presenta un proceso posterior distal en la tibia en forma de navaja (Etheridge 1995). Este proceso está asociado a la hipertrofia del músculo tibialis anticus (Abdala et al. 2006). Dentro de este grupo, se asigna a la serie de L. montanus, ya que presenta escamas de igual 


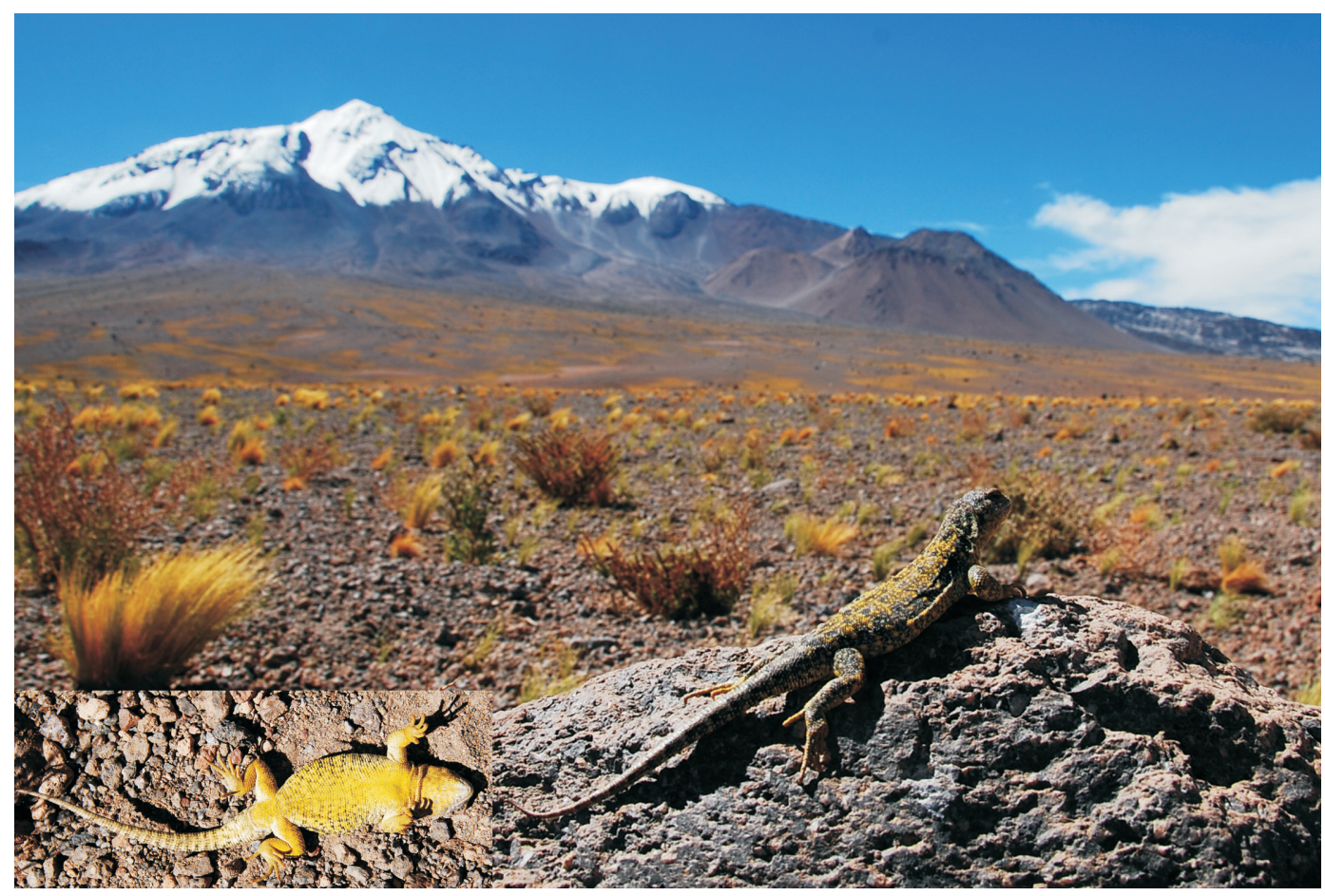

Fig. 2. Vista dorsal y ventral de un macho de Liolaemus porosus sp. nov.

Fig. 2. Dorsal and ventral views of a male Liolaemus porosus sp. nov.

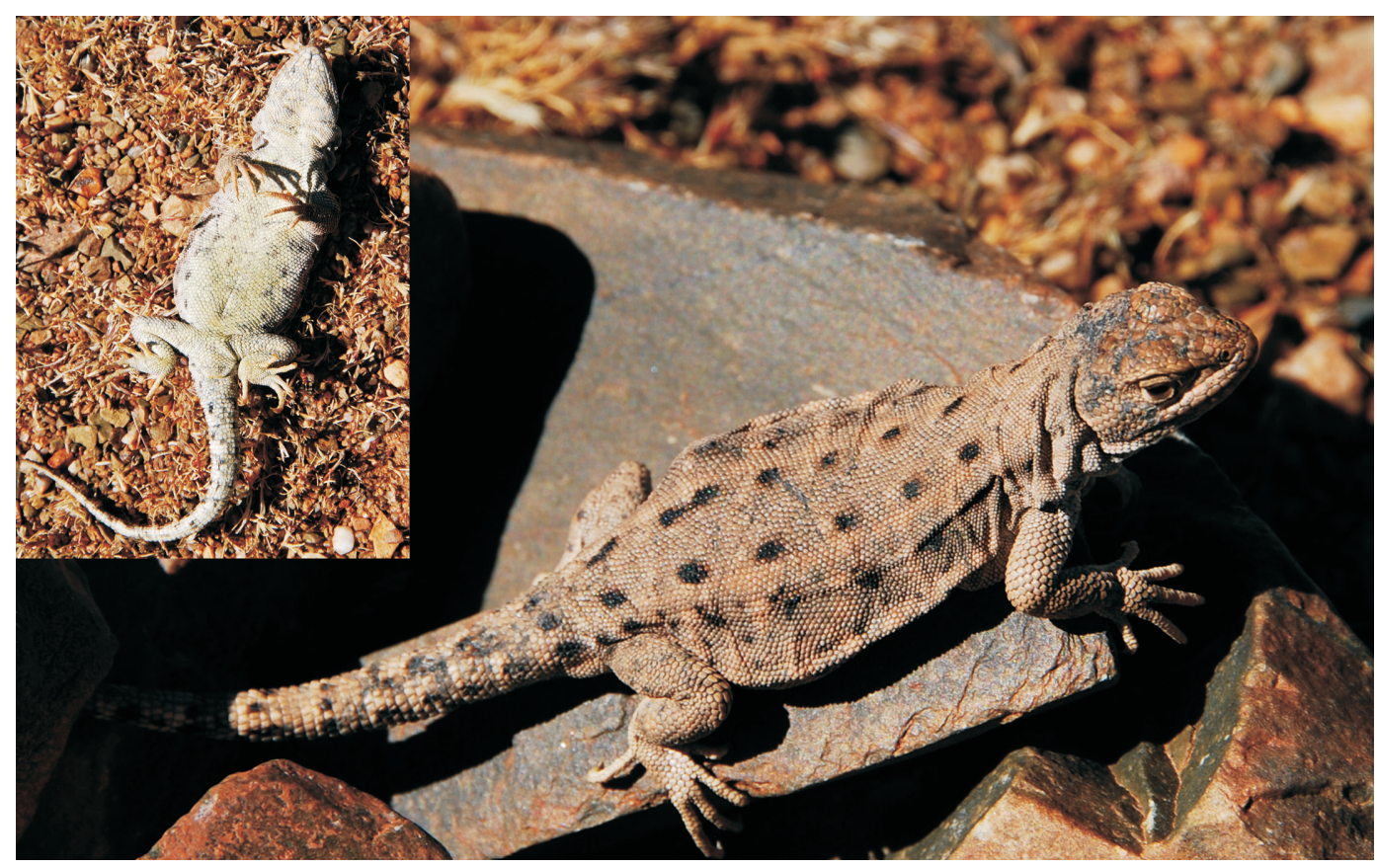

Fig. 3. Vista dorsal y ventral de una hembra de Liolaemus porosus sp. nov.

Fig. 3. Dorsal and ventral views of a female Liolaemus porosus sp. nov. 
tamaño en la parte posterior del muslo, diferenciándose de las especies del grupo de $L$. boulengeri (Avila et al. 2006, Abdala 2007) o serie boulengeri que tiene la cara posterior del músculo con parche de escamas agrandadas o diferenciadas (Schulte et al. 2000). Liolaemus porosus sp. nov. es un Liolaemus de pequeño tamaño (Cuadro 1 y 2). La superficie dorsal de la cabeza es lisa al igual que las escamas temporales. Las escamas en el cuello, tienen forma granular, sin quilla. Los pliegues del cuello están bien desarrollados. Tiene escamas antehumerales laminares, sin quilla, imbricadas, diferenciadas del resto. Las escamas gulares

\section{CUADRO 1}

Datos morfométricos y merísticos de Liolaemus porosus sp. nov.

TABLE 1

Morphometric and meristic data of Liolaemus porosus sp. nov.

\begin{tabular}{|c|c|c|}
\hline Caracteres & Holotipo & Paratipos \\
\hline Longitud hocico-cloaca & 72.9 & $64.6-72.9(67.3)$ \\
\hline Largo de la cabeza & 17.1 & $13.4-17.1(15.2)$ \\
\hline Ancho de la cabeza & 15.0 & $11.9-15.0(13.5)$ \\
\hline Alto de la cabeza & 9.8 & $7.4-9.9(8.6)$ \\
\hline Alto del oído & 2.6 & $2.1-2.8(2.4)$ \\
\hline Ancho del oído & 0.8 & $0.7-1.3(1.0)$ \\
\hline Longitud del torso & 33.3 & $28.9-33.3(31.3)$ \\
\hline Ancho del torso & 24.7 & $20.5-24.8(22.6)$ \\
\hline Longitud de la cola & 79.1 & $67.3-81.8(74.5)$ \\
\hline Número de escamas entre la rostral y el occipucio & 20 & $16-21(18.5)$ \\
\hline Número de escamas entre la rostral y la frontal & 8 & $7-10(8.1)$ \\
\hline Número de escamas entre la nasal y la cantal & 2 & $2-3(2.1)$ \\
\hline Número de escamas que rodean la nasal & 8 & $6-8(6.9)$ \\
\hline Número de escamas que rodean la interparietal & 9 & $5-9(7.6)$ \\
\hline Número de escamas supraoculares & 6 & $4-7(5.6)$ \\
\hline Número de escamas temporales & 10 & $10-12(10.6)$ \\
\hline Número de escamas superciliares & 4 & $4-5(4.4)$ \\
\hline Número de escamas auriculares & 1 & $1-3(1.8)$ \\
\hline Número de escamas ciliares superiores & 13 & $9-13(11.3)$ \\
\hline Número de escamas loreolabiales & 9 & $6-9(7.4)$ \\
\hline Número de escamas supralabiales & 10 & $7-10(8.8)$ \\
\hline Número de escamas infralabiales & 6 & $5-7(5.7)$ \\
\hline Número de escamas escamas en el cuello & 50 & $47-51(49.0)$ \\
\hline Número de escamas gulares & 52 & $41-52(46.1)$ \\
\hline Número de escamas alrededor del cuerpo & 94 & $74-96(88.0)$ \\
\hline Número de escamas dorsales del cuerpo & 95 & 89-104 (95.6) \\
\hline Número de escamas escamas ventrales & 106 & $92-109(99.8)$ \\
\hline Número de poros precloacales en machos & 6 & $5-7(5.8)$ \\
\hline Número de poros precloacales en hembras & - & $4-5(4.4)$ \\
\hline Número de poros supernumerarios en machos & 12 & $1-12(5.5)$ \\
\hline Número de poros postcloacales en machos & 8 & $1-8(3.6)$ \\
\hline Número de laminillas infradigitales IV dedo de la mano & 19 & $18-22(19.9)$ \\
\hline Número de laminillas infradigitales IV dedo del pie & 24 & $21-27(24.9)$ \\
\hline
\end{tabular}

Promedio entre paréntesis, valores de longitud en mm. Average in parenthesis, length values in $\mathrm{mm}$. 


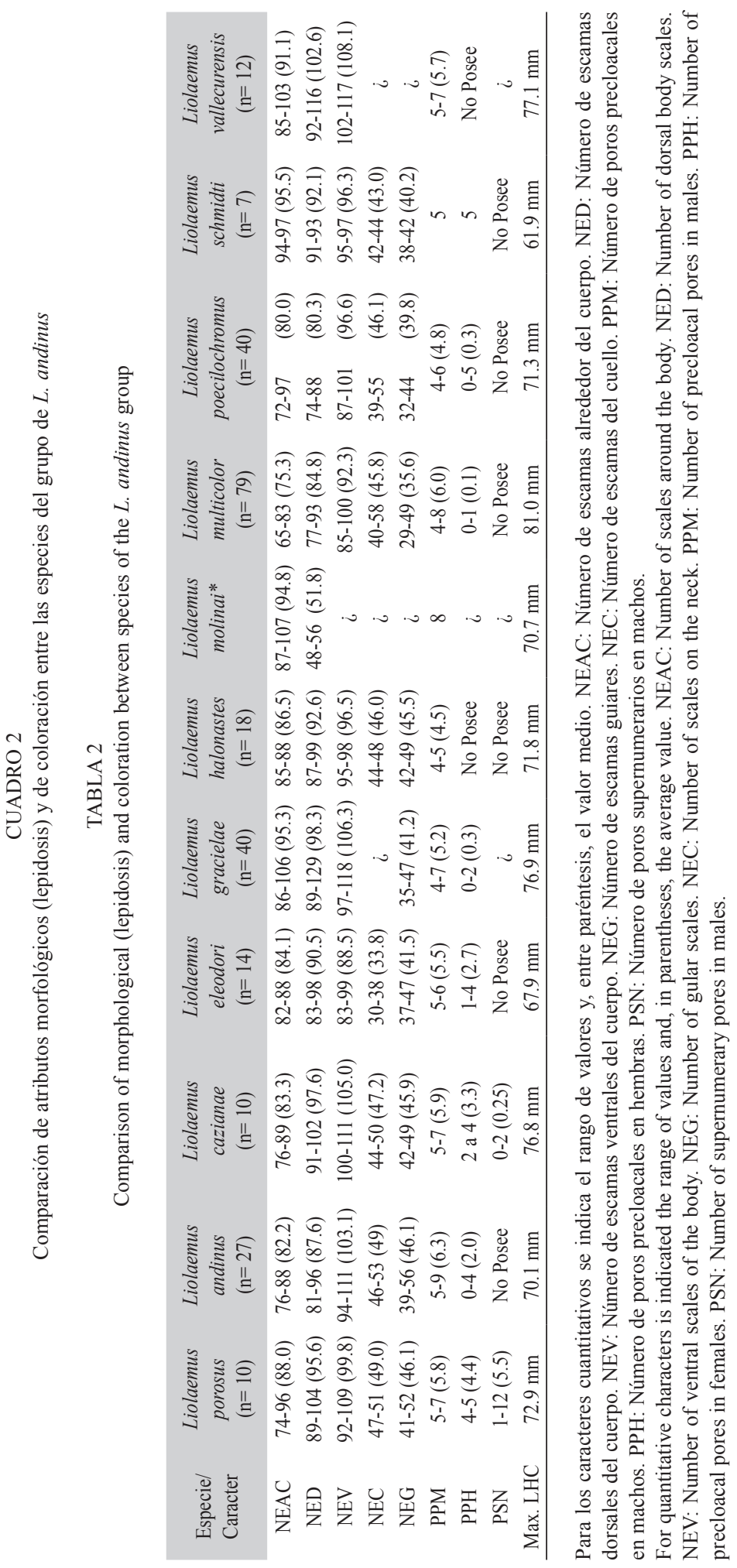


son laminares, lisas, e imbricadas. Con pliegue gular marcado, en algunos ejemplares discontinuo en el centro de la garganta. Las escamas nucales centrales se disponen en forma de cono y son granulares, sin quilla, mientras que las nucales laterales se disponen de igual forma que las centrales pero son menos cónicas. Las escamas dorsales del cuerpo son laminares cónicas, sin quilla, sin mucrón, yuxtapuestas o subyuxtapuestas. Las escamas ventrales son un poco más grandes que las dorsales. En los seis machos examinados se encontraron poros supernumerarios, dispuestos en una, dos o tres hileras, y en cinco de ellos se observaron poros post-cloacales (Fig. 4). La longitud de la cola es poco mayor que la longitud hocico-cloaca y está cubierta por escamas dorsales y ventrales laminares, imbricadas, sin quilla.

Liolaemus porosus sp. nov. posee dicromatismo sexual evidente (Fig. 2-3). En los machos, el color dorsal de la cabeza es negro o castaño claro en ejemplares de menor tamaño, siempre con numerosas escamas y manchas de color blanco o gris claro distribuidas irregularmente. Los lados de la cabeza son de color negro o gris claro.

En el cuerpo de los machos adultos, el color predominante observado es el amarillo, sin embargo el ejemplar FML 24775 es de color gris claro, presentando pequeñas manchas amarillas intercaladas entre las manchas paravertebrales. En los machos juveniles el color

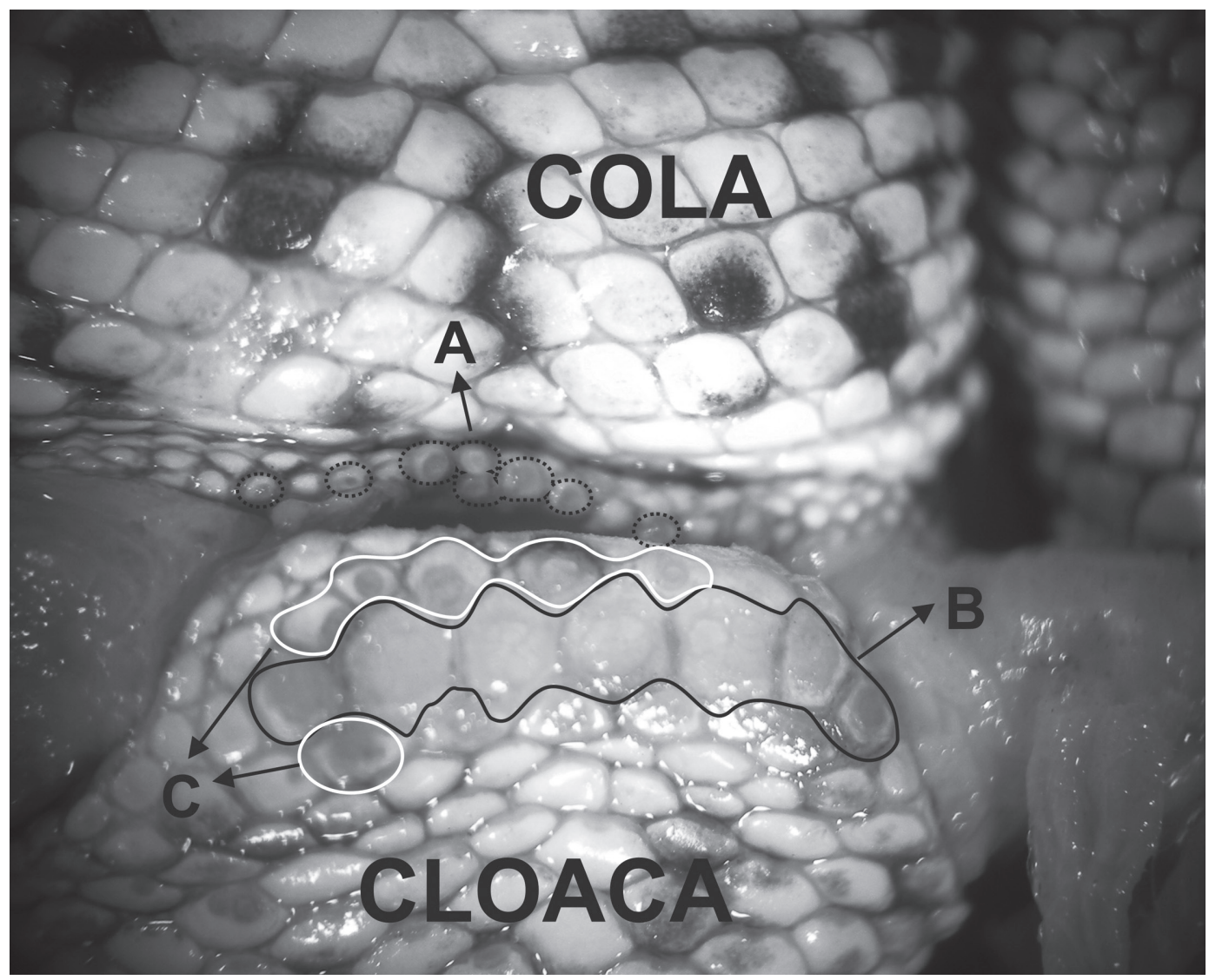

Fig. 4. Foto que exhibe los poros post-cloacales (A), poros precloacales (B) y poros supernumerarios (C) en el holotipo de Liolaemus porosus sp. nov.

Fig. 4. Photo exhibiting postcloacal pores (A), precloacal pores (B) and supernumerary pores (C) in the holotype of Liolaemus porosus sp. nov. 
del cuerpo es castaño oscuro con matices amarillentos. No se aprecia una línea vertebral definida, ni bandas dorsolaterales. Existen manchas paravertebrales y laterales de color negro, de forma subcuadrangular, variando en su tamaño. En algunos machos las manchas paravertebrales se unen con las manchas laterales formando estrías o líneas irregulares transversales al eje del cuerpo. En todos los ejemplares machos se destacan cuantiosas escamas blancas y gris claro que se esparcen sobre todo el dorso de manera irregular. Estas escamas también están en las manchas paravertebrales o laterales. Sin manchas escapulares evidentes, aunque las manchas de los lados del cuerpo llegan hasta el cuello. El cuello en sus lados es de color gris claro con varias escamas negras. Los lados del cuerpo son de color similar al del cuello, aunque se destacan manchas amarillas, irregulares pero significativas. El color dorsal de los miembros puede ser amarillo, castaño oscuro o gris, sobresaliendo algunas manchas oscuras, castañas o amarillas. El dorso de la cola es de color amarillo en su parte proximal, mientras que en el centro y región distal puede ser de color amarillo, gris o castaño claro; siempre esparcida de numerosas manchas y escamas de color negro, castaño y blanco. En general, los machos de mayor tamaño presentan mucho color amarillo en su cuerpo, mientras que en los jóvenes predomina el color castaño oscuro. El color amarillo también está presente en la región gular, pecho, vientre, cloaca, miembros anteriores y posteriores, y cola. Solo la región mental y post-mental es de color gris claro. Asimismo se acentúan cientos de pequeñas manchas negras distribuidas irregularmente en toda la región ventral de los machos.

Las hembras tienen un patrón totalmente diferente al de los machos. La cabeza es de color castaño claro con matices rojizos. El cuerpo varía de color castaño claro a gris claro, destacándose de siete a ocho pares de manchas paravertebrales. Dichas manchas son muy pequeñas, con forma de punto y de color negro. En los lados del cuerpo, miembros anteriores y posteriores, así como en la cola, se continúa el patrón dorsal del cuerpo. Ventralmente las hembras tienen un patrón similar al de los machos diferenciándose en dos aspectos: en primer lugar las manchas negras, presentes a lo largo de todo el vientre, se encuentran en menor cantidad que en los machos y son de mayor tamaño; en segundo lugar, las hembras no presentan el color amarillo característico de los machos, pero sí tienen un color verde oliva muy tenue que se acentúa en el centro de la garganta y vientre, así como en las muñecas y tobillos.

Coloración en vida del holotipo (Fig. 1): La cabeza, dorsal y lateralmente, es de color negro con numerosas escamas de color blanco y castaño oscuro, esparcidas irregularmente. $\mathrm{La}$ región occipital y cuello es de color gris claro. El cuerpo dorsalmente es de color amarillo intenso. Tiene ocho pares de manchas paravertebrales de color negro, cuyos bordes y límites son difusos; tienen forma sub-cuadrangular y se unen entre sí por intermedio de finas líneas y manchas negras en la región vertebral. Las manchas paravertebrales anteriores son pequeñas y van incrementando su tamaño hacia el centro del cuerpo. No se observa línea vertebral, bandas dorsolaterales, arco antehumeral ni manchas escapulares. Los lados del cuerpo son de color gris claro y también se destacan manchas negras de forma irregular y de mayor tamaño que las paravertebrales. Las manchas laterales del centro del cuerpo están separadas entre sí por estrías de color amarillo. Tanto el dorso como el cuello y lados del cuerpo están esparcidos de cuantiosas escamas de color blanco o gris claro. El pliegue lateral del cuerpo está formado por dichas escamas y algunas manchas amarillas que se continúan con la región ventro-lateral.

Los miembros anteriores y posteriores son de color gris claro con varias manchas negras y algunas máculas irregulares de color amarillo. Los dedos de las manos y pies son de color amarillo intenso. El dorso de la cola es de color castaño claro con manchas y escamas de color negro, blanco y algunos matices amarillentos.

Ventralmente el patrón de coloración es uniforme, se destaca el color amarillo variegado 
irregularmente de pequeñas manchas negras. Este patrón se observa en la garganta, cuello, vientre, miembros anteriores y posteriores, cloaca y parte anterior y central de la cola. La región mental y post-mental es de color gris claro, también con pequeñas manchas negras. Este mismo patrón se repite en la parte distal de la cola. El ejemplar holotipo, en estado de conservación en alcohol al 70\%, mantiene el patrón de coloración observado en vida, pero el color amarillo es menos intenso.

Distribución (Fig. 5): Esta nueva especie de Liolaemus se distribuye en un área próxima al volcán Socompa (24²8’24.1” S y $68^{\circ} 14^{\prime} 44.0$ " W), tanto en el sector limítrofe de Argentina como en Chile. En Argentina se lo encontró siempre siguiendo la ruta provincial 160 , desde el límite con Chile hasta $29 \mathrm{~km}$ al Este (243' $39.8^{\prime \prime}$ S y 68 $\left.8^{\circ} 11^{\prime} 22.6^{\prime \prime} \mathrm{W}\right)$, siempre por arriba de los $3600 \mathrm{msnm}$. En la República de Chile fue colectado en el portezuelo Socompa en 1983, a aproximadamente unos $3 \mathrm{Km}$. del límite con Argentina (24 $27^{\circ} 7.6782^{\prime \prime} \mathrm{S}$ y $68^{\circ}$ 17 ' $\left.34.728^{\prime \prime} \mathrm{W}\right)$, siguiendo la ruta 160 hacia el Oeste (Pagaburo com. pers.)

Historia natural: Liolaemus porosus habita un área montañosa, muy hostil, por arriba de los 3 600msnm (Fig. 2). En el ambiente

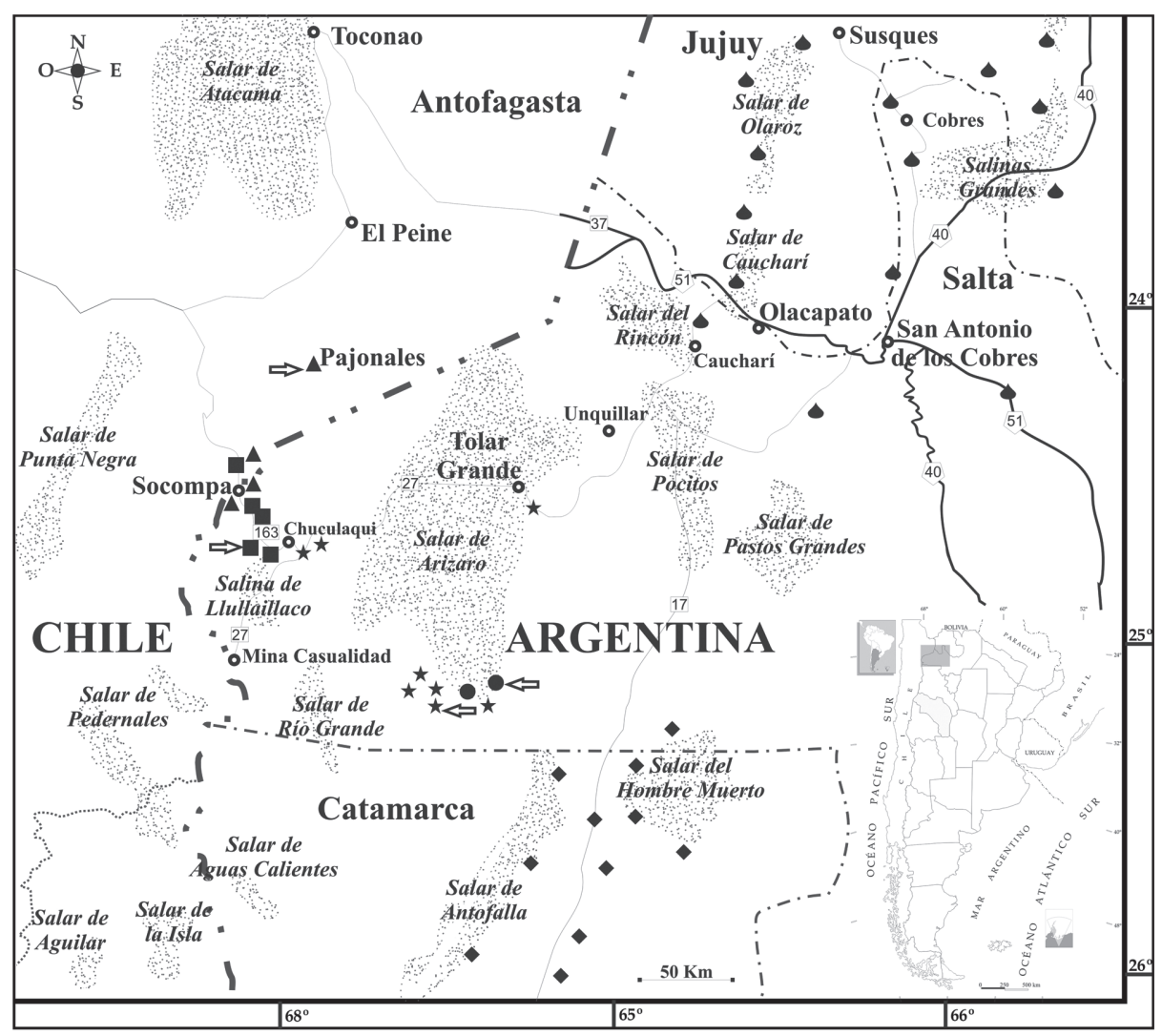

Fig. 5. Area de distribución conocida de Liolaemus porosus sp. nov. y las especies del grupo de L. montanus cercanas geográficamente. Cuadro negro: L. porosus. Triángulo negro: L. nigriceps. Estrella negra: L. cazianae. Círculo negro: L. halonastes. Rombo negro: L. poecilochromus. Lágrima negra: L. multicolor. Las flechas indican la localidad tipo.

Fig. 5. Known distribution of Liolaemus porosus sp. nov. and the closest geographically species of the L. montanus group. Black square: L. porosus. Black triangle: L. nigriceps. Black stars: L. cazianae. Black circle: L. halonastes. Black Diamond: L. poecilochromus. Black tear: L. multicolor. 
predomina un suelo arenoso entremezclado con rocas, destacándose varias cuevas construidas por roedores. La vegetación es escasa, hay pocos arbustos entre las grandes rocas volcánicas (Fig. 2). El clima es típico de alta montaña, con una marcada amplitud térmica diaria y estacional. Son comunes los fuertes vientos, así como las copiosas nevadas invernales que llegan a cubrir el suelo con más de tres metros de nieve. Los ejemplares de esta especie fueron encontrados entre las 11:00 y 15:00h, siempre asociados a rocas, las cuales utilizan para refugiarse y asolearse sobre su superficie. Los estudios realizados sobre las heces indican que se alimentan de insectos, principalmente coleópteros, algunos hemípteros y pocos vegetales, mayormente hojas. Los machos de esta especie fueron muy agresivos al ser capturados, a diferencia de las hembras que se mostraron mucho más tranquilas. No se conocen más datos sobre su biología y comportamiento. L. porosus está en simpatría con L. nigriceps.

Etimología: El nombre de esta especie es un vocablo en latín (porosus $=$ poroso) que hace referencia a la gran cantidad de poros que posee, distribuidos en pre-cloacales, supernumerarios y post-cloacales.

\section{DISCUSIÓN}

Varios son los caracteres que se pueden tener en cuenta a la hora de agrupar y separar especies en diferentes clados (número y forma de las escamas dorsales del cuerpo, dicromatismo sexual, número de poros pre-cloacales en machos y hembras, poros supernumerarios en machos, máxima longitud hocico-cloaca, presencia de heteronotos dorsales y gulares, melanismo cefálico, entre otros). Sin embargo, ésta seguirá siendo una clasificación no formal y arbitraria, con base en pocos caracteres. Este tipo de clasificación fue empleada en los Liolaemus y actualmente sigue siendo utilizada en varios grupos de este género, como el grupo de L. montanus, probablemente debido a que falta una hipótesis filogenética formal que incluya la mayoría de los taxa de este grupo.
Inicialmente Cei $(1986,1993)$ propone como clasificación no formal varios grupos dentro de los Liolaemus que habitan en Argentina, cuatro de los cuales están integrados por especies que actualmente se las considera del grupo de L. montanus propuesto por Etheridge (1995). Los grupos sugeridos por Cei (1986, 1993) son: el grupo signifer (L. dorbignyi, $L$. nigriceps y $L$. orientalis), el grupo andinus ( $L$. andinus, L. multicolor y L. poecilochromus), el grupo ruibali (L. duellmannii, L. eleodori, L. famatinae y L. ruibali) y el grupo montanus (L. griseus, L. huacahuasicus y L. montanus). Posteriormente, Schulte et al. (2000), en base a un estudio filogenético molecular, denomina serie montanus al grupo montanus de Etheridge (1995), incluyendo sólo ocho terminales que representaban al clado en estudio. PincheiraDonoso \& Núñez (2005) dividen las especies del grupo de L. montanus que habitan en Chile en cuatro grupos: el grupo jamesi (L. hajeki, L. jamesi, L. puritamensis y L. stolzmanni), el grupo fabiani (L. fabiani), el grupo ruibali (L. foxi, L. nigriceps, $L$. patriciaturrae y $L$. rosenmanni) y el grupo signifer ( $L$. andinus, L. erguetae, L. multicolor, L. pantherinus, $L$. pleopholis y L. signifer). Esta división, al igual que la propuesta por Cei (1986, 1993), es parcial y parafilética, debido a que excluye a las especies que habitan en los países limítrofes (Argentina, Bolivia y Perú).

Posteriormente, Díaz Gómez (2007a, 2007b) propone el subgrupo de L. dorbignyi como integrante del grupo de L. montanus, constituido por lagartijas de gran tamaño, sin dicromatismo sexual evidente y con bajo número de escamas alrededor del cuerpo. Recientemente, Lobo et al. (2010b) también proponen dentro del grupo de L. montanus al grupo de L. andinus, sobre la base de dos sinapomorfías (alto número de escamas alrededor del cuerpo y dicromatismo sexual evidente). Lobo et al. (2010b) incluyen dentro de este grupo a L. andinus, L. duellmani, L. eleodori, L. erguetae, L. famatinae, L. foxi, L. griseus, L. huacahuasicus, L. manueli, L. molinai, L. multicolor, L. nigriceps, L. orko, L. patriciaiturrae, L. pleopholis, L. poconchiliensis, L. 
poecilochromus, L. pulcherrimus, L. robertoi, L. rosenmani, y L. vallecurensis, aunque manifiestan que a pesar de que $L$. poconchilensis, $L$. ruibali, L. famatinae, L. griseus, L. huacahuasicus, y L. orko tienen bajo número de escamas alrededor del cuerpo, habrían adquirido el dicromatismo sexual temprano en la evolución del grupo de L. montanus.

Sobre la base de las características morfológicas observadas en L. porosus sp. nov. y teniendo en cuenta las divisiones para el grupo de L. montanus mencionadas anteriormente, el nuevo taxón descripto en este trabajo sería afín al grupo de L. andinus propuesto por Cei (1986, 1993), equivalente a los grupos de L. andinus de Lobo et al. (2010b) y L. signifer de Pincheira-Donoso \& Núñez (2005). Liolaemus porosus sp. nov. presenta las dos sinapomorfías propuestas para el grupo de $L$. andinus de Lobo et al. (2010b), las cuales son el dicromatismo sexual acentuado y el elevado número de escamas alrededor del cuerpo. Sin embargo, teniendo en cuenta los resultados obtenidos para describir este nuevo taxón, los cuales se expresan claramente en la diagnosis, podemos concluir que hay varias especies con características morfométricas y de escamación, análogas a las de L. porosus. Este conjunto de especies tienen como características: dicromatismo sexual evidente; longitud máxima hocico-cloaca de hasta $75 \mathrm{~mm}$, alto número de escamas alrededor del cuerpo (desde 70 hasta más de 100), alto número de escamas dorsales entre el occipucio y miembros posteriores (más de 89), alto número de escamas ventrales (más de 90), y las escamas dorsales del cuerpo yuxtapuestas, sin quilla. Las especies que comparten dichos caracteres son: L. andinus, L. cazianae, L. porosus, L. eleodori, L. gracielae, L. halonastes, L. molinai, L. multicolor, L. poecilochromus, L. schmidti y L. vallecurensis. Nuestra propuesta es continuar con la denominación inicial de Cei (1993), redefinida por Lobo et al. (2010b), llamando a este conjunto de taxa como grupo de $L$. andinus.

Las especies del grupo de L. andinus aquí planteado tienen hembras con poros precloacales, excepto en L. halonastes, L. molinai, y $L$. robertoi, que podrían haber perdido este atributo secundariamente. Asimismo, la presencia de poros supernumerarios en los machos de $L$. porosus es un carácter exclusivo de pocas especies del grupo de L. montanus. Hasta el momento no se ha reportado este carácter para ninguna especie integrante de otros grupos de Liolaemus; incluso dentro del grupo de L. montanus aproximadamente el $20 \%$ de las especies tendrían esta particularidad, y si puntualizamos en el grupo de L. andinus sólo L. cazianae tiene machos con poros supernumerarios. Si bien $L$. cazianae y L. porosus no son simpátricas, se encuentran distribuidas muy próximas entre sí (15 a 30km de distancia), habitando un área con similares características climáticas. Los factores de presión externa pueden haber influido en la presencia de mayor cantidad de poros precloacales (Pincheira-Donoso et al. 2008). Escobar et al. (2001) sostienen que los lagartos que viven en climas extremos desarrollan un mayor número de glándulas para producir más cantidad de secreciones, probablemente de mejor calidad y duración, en el sustrato donde son liberadas. Esto se debería a que los fuertes vientos, la marcada amplitud térmica y demás aspectos rigurosos del clima del lugar que habitan, afectan negativamente a esas señales químicas en lo referido a su permanencia en el medio. Por lo tanto, aquí el factor de selección es el ambiente, que favorece a los ejemplares más efectivos en transmitir señales, en vez de la selección sexual. Esta hipótesis se adecua al hábitat extremo y el alto número de poros super-numerarios de L. porosus y L. cazianae. Sin embargo, no ha sido corroborada experimentalmente, siendo la propuesta más aceptada la de Pincheira-Donoso et al. (2008) que establece que el número de poros es una característica estrechamente relacionada a la filogenia y no a las presiones selectivas ambientales. Otro carácter morfológico que deberá ser revisado minuciosamente en las demás especies de Liolaemus es la presencia de poros post-cloacales o poros caudales (presentes en L. porosus). Dicho atributo no se ha reportado en ninguna otra especie de Liolaemidae ni de Reptilia (Cei 1986, 1993, Duellman 1979, Pough et al. 
2003, Vitt \& Caldwell 2008, Vidal-Maldonado \& Labra-Lillo 2008); por lo tanto, representa una novedad evolutiva muy particular. Debido a que este carácter no se ha encontrado en ninguna otra especie de reptil, es prematuro y aventurado plantear una hipótesis sobre su función y origen.

Es imprescindible realizar un estudio filogenético formal que incluya todas las especies del grupo de Liolaemus montanus, utilizando la mayor cantidad posible de caracteres para lograr analizar la posición filogenética de esta nueva especie dentro del grupo de L. montanus, así como las relaciones de parentesco entre las mismas.

\section{AGRADECIMIENTOS}

Agradecemos al grupo de gendarmes de Estación Socompa y a la municipalidad de Tolar Grande por la hospitalidad y ayuda brindada. A Esteban Lavilla, Sonia Kretzschmar, Alejandro Laspiur, y especialmente a Jorge Williams por el préstamo de ejemplares holotipos y paratipos. A las Direcciones de Fauna de Catamarca, La Rioja y Salta por permitir realizar los trabajos y colectas de campo. A Pablo Valladares, Jaime Troncoso y Roberto Langstroth por el valioso aporte en la elaboración del manuscrito. A Esteban Lavilla por los consejos en la elección del nombre de la nueva especie. A J. Abdala, J. C. Acosta, S. Barrionuevo, A. Laspiur, S. Quinteros, G. Scrocchi, por su invalorable ayuda en el campo. Esta fue posible realizarla gracias a la financiación de los siguientes proyectos: PICT 2010-2263 y PIP-CONICET N ${ }^{\circ} 2422$.

\section{RESUMEN}

Liolaemus, es el segundo género más diverso dentro de Iguania, con más de 245 especies. Se describe una nueva especie de Liolaemus, perteneciente al subgénero Eulaemus, grupo o sección de Liolaemus montanus. Esta especie habita en un sector cordillerano muy restringido de la Provincia de Salta, Argentina, y en la provincia de Atacama, Chile; siempre por arriba de los $3600 \mathrm{msnm}$. Este nuevo taxón presenta características de lepidosis y patrón de coloración claramente diferente a los de las demás especies que integran el grupo de L. montanus, se destaca como carácter exclusivo entre todos los Liolaemus, la presencia de poros postcloacales. Este carácter es novedoso, no sólo en el género, sino también en todos los Reptilia, tornándose un descubrimiento morfológico de suma importancia, ya que sólo se conocen para algunos reptiles únicamente poros precloacales y femorales. Con base en las observaciones de campo realizadas, este nuevo Liolaemus es una lagartija relacionada a los ambientes rocosos, que utiliza para refugiarse, es una especie omnívora y los machos son muy agresivos al ser manipulados.

Palabras clave: Liolaemus, lagartija, nueva especie, poros postcloacales, cordillera, Reptilia.

\section{REFERENCIAS}

Abdala, C. S. 2005. Una nueva especie del género Liolaemus perteneciente al complejo darwinii (Iguania: Liolaemidae) de la provincia de Catamarca, Argentina. Revista Española de Herpetología, 19, 5-17.

Abdala, C. S. 2007. Phylogeny of the boulengeri group (Iguania: Liolaemidae, Liolaemus) based on morphological and molecular characters. Zootaxa, 1538, $1-84$.

Abdala, V., Abdala, C. S. \& Tulli, M. J. 2006. Three traditional muscular characters in the phylogeny of Liolaemus (Squamata: Tropiduridae) a reappraisal. Zootaxa, 1205, 55-68.

Abdala, C. S. \& Quinteros, S. 2008. Una nueva especie de Liolaemus (Iguanidae: Liolaemini) endémica de la sierra de Fiambalá, Catamarca, Argentina. Cuadernos de Herpetología, 22, 35-47.

Abdala, C. S., Quinteros, S. \& Espinoza, R. E. 2008. Two new species of Liolaemus (Iguania: Liolaemidae) from northwestern Argentina. Herpetologica, 64, 458-471.

Abdala, C. S., Díaz Gómez, J. M. \& Juárez Heredia, V. I. 2012a. From the far reaches of Patagonia: New phylogenetic analyses and description of two new species of the Liolaemus fitzingerii clade (Iguania: Liolaemidae). Zootaxa, 3301, 34-60.

Abdala, C. S., Semhan, R. V., Moreno Azócar, D. L., Bonino, M., Paz, M. M. \& Cruz, F. 2012b. Taxonomic study and morphology based phylogeny of the patagonic clade Liolaemus melanops group (Iguania: Liolaemidae), with the description of three new taxa. Zootaxa, 3163, 1-32.

Avila, L. J., Morando, M. \& Sites Jr., J. W. 2006. Congeneric phylogeography: hypothesizing species limits and evolutionary processes in Patagonian lizards of the Liolaemus boulengeri group (Squamata: Liolaemini). Biological Journal of the Linnean. Society, 89, 241-275. 
Avila, L. J., Martinez, L. E. \& Morando, M. 2010. Lista de las lagartijas y anfisbenas de Argentina: una actualización en línea. Centro Nacional Patagónico CENPAT-CONICET. Puerto Madryn, Chubut, Argentina (Consultado: 1 Diciembre 2010, http://www. losquesevan.com)

Avila, L. J., Pérez, C. H. F., Pérez, D. R. \& Morando, M. 2011. Two new mountain lizard species of the Phymaturus genus (Squamata: Iguania) from northwestern Patagonia, Argentina. Zootaxa, 2924, 1-21.

Breitman, M. F., Parra, M., Pérez, C. H. F. \& Sites Jr, J. W. 2011. Two new species of lizards from the Liolaemus lineomaculatus section (Squamata: Iguania: Liolaemidae) from southern Patagonia. Zootaxa, 3120, 1-28.

Cei, J. M. 1986. Reptiles del centro, centro-oeste y sur de la Argentina. Herpetofauna de las zonas áridas y semiáridas. Museo Regionale di Scienze Naturali Bollettino (Torino), 4, 527.

Cei, J. M. 1993. Reptiles del noroeste, nordeste y este de la Argentina. Museo Regionale di Scienze Naturali Bollettino (Torino), 14, 949.

Díaz Gómez, J. M. 2007a. Endemism in Liolaemus (Iguania: Liolaemidae) from the Argentinian puna. South America Journal of Herpetology, 2, 59-68.

Díaz Gómez, J. M. 2007b. Reptilia, Iguania, Liolaeminae, Liolaemus, Puna, Prepuna, and mountain ranges, Northwestern Argentina. Check List, 3, 105-118.

Duellman, W. E. 1979. The South American Herpetofauna: Its origin, evolution, and dispersal. Monograph of the Museum of Natural History, University of Kansas, 7,485 .

Escobar, C. A., Labra, A. \& Niemeyer, H. M. 2001. Chemical composition of precloacal secretions of Liolaemus lizards. Journal of Chemical Ecology, 27, 1677-1690.

Espinoza, R. R., Wiens, J. J. \& Tracy, C. R. 2004. Recurrent evolution of herbivory in small, cold climate lizard: breaking the ecophysiological rules of reptilian herbivory. Proccedings of the Natural Academy of Sciences, 101, 16819-16824.

Etheridge, R. E. 1995. Redescription of Ctenoblepharys adspersa Tschudi, 1845, and the taxonomy of Liolaeminae (Reptilia: Squamata: Tropiduridae). American Museum Novitates, 3142, 1-34.

Frost, D. R. 1992. Phylogenetic analysis and taxonomy of the Tropidurus group of lizards (Iguania: Tropiduridae). American Museum Novitates, 3033, 1-68.

Ibarra-Vidal, H. 2005. Nueva especie de lagartija del altiplano de Chile, Liolaemus lopezi Sp.N. (Reptilia, Tropiduridae, Liolaeminae). Boletín de la Sociedad de Biología de Concepción, 76-77, 7-14.

Labra, A., Carazo, P., Desfilis, E. \& Font, E. 2007. Agonistic interactions in a Liolaemus lizard: structure of head bob displays. Herpetologica, 63, 11-18.
Langstroth, R. P. 2011. On the species identities of a complex Liolaemus fauna from the Altiplano and Atacama Desert: insights on Liolaemus stolzmanni, L. reichei, L. jamesi pachecoi, and L. poconchilensis (Squamata: Liolaemidae). Zootaxa, 2809, 20-32.

Laurent, R. F. 1985. Segunda contribución al conocimiento de la estructura taxonómica del género Liolaemus Wiegmann (Iguanidae). Cuadernos de Herpetología, 1, 1-37.

Laurent, R. F. 1992. On some overlooked species of the genus Liolaemus Wiegmann (Reptilia: Tropiduridae) from Peru. Breviora, 494, 1-33.

Laurent, R. F. 1998. New forms of lizards of the subgenus Eulaemus of the genus Liolaemus (Reptilia: Squamata: Tropiduridae) from Perú and northern Chile. Acta Zoológica Lilloana, 44, 1-26.

Lobo, F. 2001. A phylogenetic analysis of lizards of the Liolaemus chilensis group (Iguania: Tropiduridae). Journal of Herpetology, 11, 137-150.

Lobo, F. 2005. Las relaciones filogenéticas en el grupo chiliensis de Liolaemus (Iguania: Liolaemidae). Sumando nuevos caracteres y taxa. Acta Zoológica Lilloana, 49, 67-89.

Lobo, F. \& Espinoza, R. E. 1999. Two new cryptic species of Liolaemus (Iguania: Tropiduridae) from northwestern Argentina: resolution of the purported reproductive bimodality of Liolaemus alticolor. Copeia, 1999, 122-140.

Lobo, F., Espinoza, R. E. \& Quinteros, S. 2010a. A critical review and systematic discussion of recent classification proposals for liolaemid lizards. Zootaxa, 2549, 1-30.

Lobo, F., Slodki, D. \& Valdecantos, S. 2010b. Two New Species of Lizards of the Liolaemus montanus Group (Iguania: Liolaemidae) from the Northwestern Uplands of Argentina. Journal of Herpetology, 44, 279-293.

Martori, R. \& Aun, L. 2010. Reproducción y variación de grupos de tamaño en una población de Liolaemus koslowskyi (Squamata: Liolaemini). Cuadernos de Herpetología, 24, 39-55.

Martori, R., Aun, L. \& Rocha, C. 1999. Variación estacional de la dieta de Liolaemus wiegmanni (Squamata: Tropiduridae) en un agroecosistema del sur de Córdoba, Argentina. Cuadernos de Herpetología, 13, 69-80.

Navarro, J. \& Núñez, H. 1993. Liolaemus patriciaiturrae and Liolaemus isabelae, two new species of lizards for northern Chile: Biogeographic and cytotaxonomic aspects (Squamata, Tropiduridae). Boletín del Museo Nacional de Historia Natural, Santiago de Chile, 44, 99-113.

Núñez, H., Navarro, J., Garín, C., Pincheira-Donoso, D. \& Meriggio, V. 2003. Phrynosaura manueli y 
Phrynosaura torresi, nuevas especies de lagartijas para el norte de Chile (Squamata: Sauria). Boletín del Museo Nacional de Historia Natural, Santiago de Chile, 52, 67-88.

Núñez, H. \& Yánez, J. 1983. Ctenoblepharis audituvelatus new species, a lizard from northern Chile (Reptilia Iguanidae). Copeia, 2, 454-457.

Pincheira-Donoso, D. \& Nuñez, H. 2005. Las especies chilenas del género Liolaemus Wiegmann, 1834 (Iguania: Tropiduridae: Liolaeminae). Taxonomía, Sistemática y Evolución. Publicación Ocasional del Museo Nacional de Historia Natural (Chile), 59, 7-486.

Pincheira-Donoso, D., Scolaro, J. A. \& Sura, P. 2008. A monographic catalogue on the systematics and phylogeny of the South American iguanian lizard family Liolaemidae (Squamata, Iguania). Zootaxa, 1800, 1-85.

Pough, F. H., Andrews, R. M., Cadle, J. E., Crump, M. L., Savitsky, A. H. \& Wells, K. D. 2003. Herpetology. New Jersey, USA: Prent. Hall.

Quinteros, A. S., Abdala, C. S. \& Lobo, F. J. 2008. Redescription of Liolaemus dorbignyi Koslowsky, 1898 and description of a new species of Liolaemus (Iguania: Liolaemidae). Zootaxa, 1717, 51-67.

Quinteros, A. S. \& Abdala, C. S. 2011. A new species of Liolaemus of the Liolaemus montanus section (Iguania: Liolaemidae) from Northwestern Argentina. Zootaxa, 2789, 35-48.

Ramírez-Leyton, G. \& Pincheira-Donoso, D. 2005. Fauna del Altiplano y Desierto de Atacama. Argentina: Phrynosaura Ediciones.
Ramírez-Pinilla, M. P. 1991. Estudio histológico de los tractos reproductivos y actividad cíclica anual reproductiva de machos y hembras de dos especies del género Liolaemus (Reptilia: Sauria: Iguanidae). (Tesis Doctoral). Universidad Nacional de Tucumán, Tucumán, Argentina.

Schulte, J. A., Macey, J. R., Espinoza, R. E. \& Larson, A. 2000. Phylogenetic relationships in the iguanid lizard genus Liolaemus: multiple origins of viviparous reproduction and evidence for recurring Andean vicariance and dispersal. Biological Journal of the Linnean Society, 69, 75-102.

Valladares, J. P., Etheridge, R., Schulte, J. A., Manriquez, G. \& Spotorno, A. 2002. Nueva especie de lagartija del norte de Chile, Liolaemus molinai (Reptilia: Liolaeminae). Revista Chilena de Historia Natural, 75, 473-489.

Valladares, P. 2004. Nueva especie de lagarto del género Liolaemus (Reptilia: Liolaemidae) del norte de Chile, previamente confundido con Liolaemus (=Phrynosaura) reichei. Cuadernos de Herpetología, 18, 43-53.

Valladares, P. 2011. Análisis, síntesis y evaluación de la literatura de lagartos de la Región de Atacama, Chile. Gayana, 75, 81-98.

Vidal Maldonado, M. A. \& Labra Lillo, A. 2008. Herpetología de Chile. Santiago de Chile: Science Verlag.

Vitt, L. J. \& Caldwell, J. P. 2008. Herpetology, Third Edition: An Introductory Biology of Amphibians and Reptiles. New York, USA: Academic Press (Elsevier). 


\section{APÉNDICE I}

Listado de especímenes examinados. Los números entre paréntesis a continuación del nombre de cada taxón indican el número de individuos examinados. Obsérvese que algunos números de FML representan lotes de más de un individuo. En tales casos, el número de individuos por lote es indicado entre corchetes a continuación del número de colección.

Acrónimos: FML: Fundación Miguel Lillo. MCN: Museo de Ciencias Naturales, Universidad Nacional de Salta. SDSU: San Diego State University. MLP.S: Museo de la Plata. KU: The Museum of Natural History at the University of Kansas. UNSJ: Universidad Nacional de San Juan. CBF: Colección Boliviana de Fauna. MACN: Museo Argentino de Ciencias Naturales. IMCN-UNSJ: Instituto y Museo de Ciencias Naturales, Universidad Nacional de San Juan. MVZ: University of California at Berkeley Museum of Vertebrate Zoology. LACM: Los Angeles County Museum of Natural History.

Liolaemus andinus (27).-ARGENTINA: Provincia de Catamarca: Departamento Tinogasta, Aguas Calientes: FML 1409 [17]; Las Grutas, 20km de Paso San Francisco: FML 1913, 2025; Cerca de Río Chaschuil, 42km Sureste de Paso San Francisco: FML 2545; $68 \mathrm{~km}$ al Noroeste de Río San Francisco: FML 2546; Cazadero Grande: MCN 1648-53.

L. annectens (4).-PERÚ: Provincia de Tacna: Departamento Tacna, Sierra de Tacna: FML 340; Río cruzando la ruta 150m desde Arequipa (ruta Arequipa-Puno): FML 15431-33.

L. cazianae (10).-ARGENTINA: Provincia de Salta: Departamento Los Andes, $10 \mathrm{~km}$ al Oeste de Cauchari, y $2 \mathrm{~km}$ de Laguna Seca $\left(24^{\circ} 12^{\prime} 07.1^{\prime \prime}\right.$ S y 66 54 '39.7” W): FML 24753-55; Cuesta de Caipe camino a Socompa

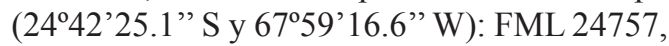
24759-60; Ojo de Mar, cerca de Tolar Grande $\left(24^{\circ} 35^{\prime} 40.2^{\prime \prime} \mathrm{S}\right.$ y $\left.67^{\circ} 22^{\prime} 50.0^{\prime \prime} \mathrm{W}\right)$ : FML 2476162; $10 \mathrm{~km}$ al Este de Tolar Grande (2439'37.5" S y $67^{\circ} 18^{\prime} 15.3$ " W): FML $24763 ; 15 \mathrm{~km}$ al
Oeste de Pocitos $\left(24^{\circ} 34^{\prime} 58.0^{\prime \prime} \mathrm{S}\right.$ y $67^{\circ} 05^{\prime} 23.0^{\prime \prime}$ W): FML 24764.

L. chlorostictus (25).-ARGENTINA: Provincia de Jujuy: Departamento Rinconada, Cuesta de Fundiciones, Mina Pirquitas: FML 1515 [14]; Laguna Vilama: FML 2706 [2]; Cuesta de Fundiciones, $47 \mathrm{~km}$ al Noroeste de Mina Pirquitas: SDSU 1613-18; $15.5 \mathrm{~km}$ al Este de Orosmayo, sobre Ruta Provincial 70 $\left(22^{\circ} 29^{\prime} 26.7^{\prime \prime}\right.$ S y $66^{\circ} 16^{\prime} 16.6$ " W): SDSU 3517-19.

L. disjunctus (3).-PERÚ: Huamachuco: FML 12011 (holotipo), 12012-13.

L. dorbignyi (24).-ARGENTINA: Provincia de Catamarca: Cordillera de Catamarca: MLP.S 57 (lectotipo), 844-45, 991-92 (paralectotipos); Departamento Tinogasta, Puesto la Lagunita, $35-38 \mathrm{~km}$ al Noreste de Medanitos, subiendo desde Medanitos, Sierra de Fiambalá: MCN 2119-21; Los Aujeritos, 34-36km al Noreste de Medanitos, $2 \mathrm{~km}$ al Suroeste de Puesto la Lagunita, Subiendo desde Medanitos, Sierra de Fiambalá: MCN 2132-33; Saujil, Ruta 60: FML 1855 [7]; Puesto la Lagunita, $35-38 \mathrm{~km}$ Noreste de Medanitos, subiendo desde Medanitos, Sierra de Fiambalá: FML 17230-36.

L. duellmani (6).-ARGENTINA: Provincia de Mendoza: Departamento Malargüe, Paso el Choique, $50 \mathrm{~km}$ al Sursuroeste de El Manzano: KU 161127-28 (paratipos); 48km al Sur de la intersección de Ruta Provincial 221 y Ruta Nacional 40 sobre Ruta 221 $\left(36^{\circ} 21^{\prime} 12.0^{\prime \prime} \mathrm{S}\right.$ y $\left.69^{\circ} 48^{\prime} 08.7^{\prime \prime} \mathrm{W}\right)$ : SDSU $3389-91 ; 19 \mathrm{~km}$ al Norte de la intersección de 
Ruta Provincial 221 y Ruta Nacional 40 sobre Ruta 221 (36²1'12.0” S y 6948'8.7” W): SDSU 3424.

L. eleodori (14).-ARGENTINA: Provincia de San Juan: Departamento Iglesia, Llano de los Hoyos, Río San Guillermo: FML 1703 [4]; Llano de los Hoyos, Cordillera de San Guillermo: SDSU 1610. Reserva Provincial y de la Biosfera San Guillermo, Caserones: UNSJ nueve ejemplares sin número.

L. erguetae (2).-BOLIVIA: Departamento Potosi, Laguna Colorada (22 13 '00.6" S y 6744'01.0” W): FML 3207-08 (paratipos).

L. etheridgei (1).-PERÚ: Departamento Arequipa: Provincia de Arequipa, 3km Noreste de Yura: SDSU 1622.

L. famatinae (35).-ARGENTINA: Provincia de La Rioja: Departamento Famatina, Cerro de Famatina: FML 232 [2]; Cueva de Perez, Nevados de Famatina: FML 1720 [32], SDSU 1624 (paratipo).

L. fittkaui (5).-BOLIVIA, Departamento Cochabamba, Tiraque: FML 16121-2. Cochabamba: CBF 2250, 2247.

L. forsteri (3).-BOLIVIA: Departamento La Paz, Chacaltay: FML 2211. La Paz: CBF 2721, 1056.

L. gracielae (40).-ARGENTINA: Provincia de la Rioja: Departamento General Sarmiento, camino a Mula Muerta, $2 \mathrm{~km}$ al Oeste de Laguna Brava, Reserva Natural Laguna Brava (2816'10.4" S y 68 $51^{\prime} 26.0^{\prime \prime}$ W, 4 300m): FML 17866; FML 17861-65, 1786780; 18346-55. Provincia de San Juan: Departamento Iglesia, Llano Negro, $6 \mathrm{~km}$ al Norte del río Santa Rosa, Reserva Provincial y Reserva de la Biosfera San Guillermo (28 56'12.3" S y 69¹5'31.6” W): IMCN-UNSJ 4130-39.

L. griseus (9).-ARGENTINA: Provincia de Tucumán: Departamento Tafí del Valle,
Vega Mataderos, Vacahuasi: FML 1354 [4]; Suroeste de Cerro Lomo Ballena, Cumbres Calchaquíes: FML 1582; Cerro Negrito, Cumbres Calchaquíes: FML 1586 [4].

L. halonastes (18).-ARGENTINA: Provincia de Salta: Departamento Los Andes, Extremo Sur del Salar de Arizaro (2504'34.2" S y $67^{\circ} 40$ '01.1" W, 3 516msnm): FML 2473435; Margenes del Salar de Arizaro a $11 \mathrm{~km}$ al suroeste del Campamento Mina Arita, ( $25^{\circ} 04.5709 \mathrm{~S}$ y $\left.67^{\circ} 40.0199 \mathrm{~W}, 3491 \mathrm{msnm}\right)$ : MACN 39216 (Holotipo). Mismos datos que el holotipo: MACN 39217-39218; MCN 25232524, 2556, 2558-2567, 2569.

L. huacahuasicus (39).-ARGENTINA: Provincia de Catamarca: Departamento Andalgalá, Filo los Heladitos, Subiendo desde Mina Capillitas: FML 1224 [18]; Departamento Santa María, El Cerrillo: FML 486-87; $1 \mathrm{~km}$ al Sur de Cerro El Overo, Nevados del Aconquija: FML 665; Filo Colorado, dos a $3 \mathrm{~km}$ al Oeste de Cerro El Overo, Nevados del Aconquija: FML 674-75; $5 \mathrm{~km}$ al Oeste de Nevado del Candado: FML 677; Nevado de las Animas: FML 885 [7]; Provincia de Tucumán: Departamento Tafí del Valle, Laguna de El Negrito: FML 66 [3] (paratipos); Cerro El Negrito: FML 203 [2], 469-70 (paratipos), SDSU 1623.

L. huayra (9).-ARGENTINA: Provincia de Tucumán: Departamento Tafí del Valle, Cerro el Pichao, Sierra de Quilmes (26²2'26.7'" S y $\left.66^{\circ} 04^{\prime} 54.2^{\prime \prime} \mathrm{W}, 3545 \mathrm{msnm}\right)$ : FML 18069 (holotipo), MCN 2184 (paratipo); Puesto Pacheco, Subiendo desde Pichao, Sierra de Quilmes: FML 1219 [7] (paratipos).

L. inti (13).-ARGENTINA: Provincia de Salta: Departamento Cachi, Cerca de la $15^{\circ}$ Estación del Vía Crucis, Cerro de la Virgen,

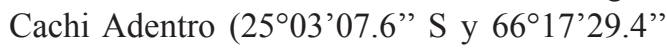
W, 3 938msnm): FML 18399 (holotipo), MCN 2183 (paratipo); Cerro de la Virgen, Cachi Adentro: FML 1669 [7] (paratipos); Mina Santa Elena, Nevados de Palermo (4 000m): 
FML 1432 [2]; Ladera Suroeste de Nevado de Cachi (3 500msnm): FML 9652 [2].

L. islugensis (4).-BOLIVIA: Departamento de Tarija: CBF 1778-9, 1789-90.

L. jamesi (5).-CHILE, Región I, Tarapacá: Punta Arica: FML 1193; Parinacota, Arica: FML 1775 [2]. Región II, Antofagasta: Alta Camina, $177 \mathrm{~km}$ al Noreste de Iquique: MVZ 66805-06.

L. melanogaster (2).-PERÚ: Departamento de Ayacucho, $45 \mathrm{~km}$ al Este de Puquio (14울' S y $74^{\circ} 08^{\prime} \mathrm{W}$ ): FML 2491 [2] (paratipos).

L. montanus (36).-ARGENTINA: Provincia de Catamarca: Departamento Ambato, El Rodeo: FML 279 [7], 289; Cerro El Manchao: FML 908-10, 981-82, 1723 [16], 2141 [5]; Falda, Base Este de Cerro El Manchao: SDSU 1621.

L. multicolor (79).-ARGENTINA: Provincia de Jujuy: Departamento Cochinota, Abra Pampa: FML 259, 272 [3], 1873 [9], 2065 [18], 2098 [4], 2349 [2], 2592; 3km Noroeste de Abra Pampa: FML 1464 [13], 1539 [16]; 3km Noroeste de Abra Pampa sobre Ruta Provincial 7: SDSU 1314-19; $4.2 \mathrm{~km}$ al Oeste de Abra Pampa sobre Ruta Provincial 71 (2242'24.4" S y 6543'12.4" W): SDSU 3589-91; Provincia de Salta: Departamento Los Andes, $11.1 \mathrm{~km}$ al Este de Olacapato sobre Ruta Nacional 51 (2410'10.9" S y 66³9'05.8" W): SDSU 3592, 3595-96.

L. porosus (10).-ARGENTINA: Provincia de Salta: Departamento Los Andes, $23 \mathrm{~km}$ de Gendarmería Socompa (243' $40.2^{\prime}$ ' S, y 6811'58.4"W, 3 707msnm), FML 24771 (holotipo); Pie Este del Volcán Socompa (242'24.1" S y 68 14'44.0" W): FML 24780 (paratipo); A $20 \mathrm{~km}$ de Gendarmería Socompa (243' 40.2" S y 68 $11^{\circ}$ '58.4" W, $3707 \mathrm{msnm}$ ): FML 24773 (paratipo); $13 \mathrm{~km}$ al Noroeste de Chuculaqui (2440'17.3" S y 68 09'18.5" W, 4 209msnm): FML 24774 (paratipo); 23km de Gendarmería Socompa (24³6'16.5" S y 68'12'33.1" W, 3 861msnm): FML 24772 (paratipo); $29 \mathrm{~km}$ de Gendarmería Socompa (243'39.8" S y 68¹1'22.6" W, 3 884msnm): FML 24778-9 (paratipos). $6.5 \mathrm{~km}$ de Gendarmería Socompa (242 $28^{\prime} 43.8^{\prime \prime} \mathrm{S}$ y $68^{\circ} 14^{\prime} 15.9^{\prime \prime}$ W, 3 641msnm): FML 24775-77; CHILE: Provincia de Antofagasta: Portezuelo Socompa, $\left(24^{\circ} 27^{\prime} 7.6782^{\prime \prime}\right.$ S y $68^{\circ} 17^{\prime} 34.728^{\prime}$ W): FML 01647 .

L. nigriceps (76).-ARGENTINA: Provincia de Salta: Departamento Los Andes, Cono aluvial al Sur del Volcán Socompa: FML 1633 [3], 1635 [31]; Quebrada de ladera del cerro Socompa: FML 1632 [4]; 3km al Este estación Socompa: FML 1634 [2], SDSU 3687-88; Ladera norte del cerro al sur del Volcán Socompa: FML 1635 [21], 1680 [2]; CHILE: Región II, Antofagasta: Socompa, a $8 \mathrm{~km}$ de la frontera: FML 923-24; Portezuelo Socompa: FML 1636; Socompa: SDSU 1620; 7km de Estación Socompa $\left(24^{\circ} 28^{\prime} 14.4^{\prime \prime} \mathrm{S}\right.$ y $\left.68^{\circ} 15^{\prime} 10.7^{\prime \prime} \mathrm{W}\right)$ : FML 24717-22; $1 \mathrm{~km}$ de Gendarmería Socompa $\left(24^{\circ} 27^{\prime} 38.0\right.$ " S y $\left.68^{\circ} 15^{\prime} 10.7^{\prime \prime} \mathrm{W}\right)$ : FML 24723-27.

L. orientalis (38).-ARGENTINA: Provincia de Jujuy: Departamento Humahuaca, Camino a Laguna Blanca: FML 928 [4], 949 [2]; Chorcán: FML 930, 938-39, 944; Camino a Laguna Blanca: FML 949 [2]; Laguna Leandro, Oeste del Chorcán: FML 1456 [4]; Mina Aguilar, Exposición Suroeste FML 1537 [2]; Quebrada Tonocote: FML 2035 [14]; Tablayo, Oeste de Chaupi Rodeo: FML 2104 [6].

L. orko (13).-ARGENTINA: Provincia de Catamarca: Departamento Tinogasta, Puesto La Lagunita, Sierra de Fiambalá: FML 18416 (holotipo); FML 18417-21 (paratipos); MCN 2130-31 (paratipos); Las Pampas, Campo Potreritos: FML 1911 [5].

L. pachecoi (2).-BOLIVIA: Departamento de Potosí, Sud Lipez: CBF 1057 (holotipo), Laguna Colorada: FML 2788 (paratipo). 
L. poecilochromus (40).-ARGENTINA: Provincia de Catamarca: Departamento Antofagasta de la Sierra, Salar de Antofalla: FML 878; Los Nacimientos: FML 1171 [12] (paratipos); Volcanes de Los Nacimientos: FML 1173; Ojo de Calalaste: FML 3068 [2]; Las Quinuas, Salar de Antofalla: FML 369-71; 4-5km al Norte de Antofagasta de la Sierra: MCN 24951; 288-91; Paycuqui: MCN 278-79, 298-301; $3 \mathrm{~km}$ al Norte de Antofagasta de la Sierra: MCN 280-82; Cuesta de Randolfo: MCN 28587; departamento Tinogasta, Aguas Calientes: SDSU 1597; Provincia de Salta: Departamento Los Andes, $7 \mathrm{~km}$ al Suroeste de Huatiquina: SDSU 1598-99.

L. poconchilensis (2).-CHILE: Región I, Tarapacá: Pampa Chaca: LACM 9312; PERÚ: Departamento Tacna: Provincia Tacna, 6.4km al Norte de Tacna: MVZ 99663.

L. polystictus (2).-PERÚ: Departamento de Huancavelica, localidad de Santa Inez: FML 1683 [2] (paratipos).

\section{L. pulcherrimus (32).-ARGENTINA:} Provincia de Jujuy: Departamento Humahuaca, Mudana: FML 1961 [2], 2184 [15] (paratipos). Camino a Mudana desde Uquía (2320’30" S y 65'13'27.5” W): FML 18221, 18238-49. 24km al Este de Uquía por camino a Mudana: FML $18285,18213$.

L. puritamensis (12).-ARGENTINA: Provincia de Jujuy: Departamento Rinconada, Rocas sobre la orilla Oeste de Laguna Vilama: MCN 2498; Camino entre la intersección de la ruta a Laguna Cerro Negro y la ruta a Laguna Isla Grande: MCN 2500; Rocas sobre la orilla Este de Laguna Vilama: MCN 2505; Peñón sobre la orilla Sureste de Laguna Vilama: MCN 2507-08; Camino a Laguna Isla Grande, al Este de Vilama sobre la orilla a Cerro Granada y Mina Pirquitas: MCN 2509-13; Alrededores de Laguna Cerro Negro: MCN 2517-19.

L. robertoi (2).-CHILE: Cuarta Región Administrativa de Chile, Baños del toro, $\left(29^{\circ} 51^{\prime} 17.2\right.$ " S y $70^{\circ} 02$ '41.5” W): FML 2026 [2].

L. ruibali (52).-ARGENTINA: Provincia de Mendoza: Departamento Las Heras, Paramillo de Uspallata: FML 1076, 1104; Ruta Nacional 7, Paramillo, Uspallata: FML 2549 [7]; 24km al Sureste de Uspallata: FML 346263; $25 \mathrm{~km}$ al Oeste de Uspallata, sobre Ruta Provincial 7: FML 3730 [5]; 20km al Noreste de Uspallata: SDSU 1604-05; Provincia de San Juan: Departamento Iglesia, suelo gravoso al Sur de Tocota $\left(30^{\circ} 41^{\prime} 0.25^{\prime \prime} \mathrm{S}\right.$ y $69^{\circ} 26^{\prime} 38.1^{\prime \prime}$ W): FML 3461 [12], SDSU: 3436-39, 3454-58, 3502-14.

L. schmidti (7).-CHILE: Región II, Antofagasta: Atacama: FML 1192; Volcán Tatio: FML 1197; Ojos San Pedro, 88.5km al Noreste de Calama: MVZ 66807-08; Región I, Tarapacá: Tarapacá, Cariquima: SDSU 1601-03.

L. scrocchii (26).-ARGENTINA: Provincia de Jujuy: Departamento General Belgrano, Alrededor de la base de Cerro Paño, Serranía de Chañi: FML 992 [4] (paratipos); Casa Mocha, Subiendo desde el Noroeste de Nevado del Chañi: MCN 714, 898, 961 (paratipos); Departamento Humahuaca, Abra del Cerro Toro, Sierra de Aguilar: MCN 899 (paratipos); Departamento Susques, $2 \mathrm{~km}$ al Sureste de Susques sobre Ruta Nacional 52: FML 17228 (holotipo), 17229 (paratipo); Departamento Tumbaya, El Quemado, Abra de Pives: FML 1817-1 (paratipo); Susques Pueblo: FML 976, 978 [2]; Provincia de Salta: Departamento La Poma, La Mesada, 64km desde La Poma a Abra del Acay: FML 6481 (paratipo); Departamento Los Andes, Olacapato, Quebrada de Los Berros: 1365 [3]; Santa Rosa de los Pastos Grandes: FML 1763 (paratipo); Agua de los Sapos (Puesto de Cruz Soriano), a 20km desde Mina Tincalcayo: FML 1759 [2] (paratipos); camino a Abra del Acay desde Ruta Nacional 51: MCN 964 (paratipo); Señal del km 210, Ruta Nacional 51, 0.6km desde Ruta Nacional $51,6.4 \mathrm{~km}$ al Sur de Olacapato $\left(24.2411475^{\circ}\right.$ $\mathrm{S}$ y $66.69711^{\circ} \mathrm{W}$ ): MCN 1922-25 (paratipos). 
L. signifer (24).-BOLIVIA: Departamento Cochabamba: Provincia Tapacari, Confital, camino a la La Paz- Cochabamba: FML 1562 [2]; PERÚ : Departamento Apurimac: Provincia de Aymaraes, $40 \mathrm{~km}$ al Suroeste de Chalhuanca: MVZ 57832-33; Departamento Arequipa: Provincia de Caylloma: Vincocaya: CAS 80901-02, 80904; Departamento Ayacucho: Provincia de Lucanas, $35 \mathrm{~km}$ ENE Puquio: MVZ 57834; Departamento Moquegua: Pampa Huatire: MVZ 57811; Departamento Puno: Provincia de Puno, Lago Titicaca: FML 1434 [2]; Lago Titicaca camino a Puno: FML 1557; $82 \mathrm{~km}$ al Oeste de Puno: MVZ 57827; Pichupichuin, $8 \mathrm{~km}$ al Noroeste de Huacallani: MVZ 57830; Caccahara: MVZ 57837; 12.9km al Sursuroeste de Limbani: MVZ 57843; 4km al Nornoroeste de Juliaca: MVZ 83692; Huaylarco, 88.5km ENE Arequipa: MVZ 92871; 5km al Oeste de Tincopalca: SDSU 1600; Departamento Tacna: Provincia Tacna, $5 \mathrm{~km}$ al Este de Lago Suche: MVZ 57823; Provincia de Tarata, 16km al Sur de Tarata: MVZ 99650-53.
L. vallecurensis (12).-ARGENTINA: Provincia de San Juan: Departamento Iglesia, Valle de Cura: FML 2404. Provincia de San Juan: Departamento Iglesia, Valle de Cura: UNSJ 4242-44, 4247, 4249-50, 4253, 4257, 4260, 4265,4270 .

L. vulcanus (23).- ARGENTINA: Provincia de Catamarca, Departamento Antofagasta de la Sierra: Pocitos y Loma Cortada, Suroeste de Los Nacimientos: FML 01169 (Holotipo), FML 23819 (Paratipo); Trapiche, Tramo del Río Punilla: FML 1167 [13] (Paratipos); Río Punilla: MCN 270-271 (Paratipos); Paycuqui: MCN 273; MCN 302; MCN 304; MCN 311 (paratipos). $61 \mathrm{~km}$ al Sur de FMC $(25.83610$ S y 67.29279 W, 4 128msnm): MCN 1926-27 (paratipos).

L. willamsi (2).- PERÚ: Departamento de Ayacucho, Pampa Galeras entre Nazca y Puquio: FML 1701 (paratipo). Lucanas, Pampa Galeras a 96km de Nazca: FML 13403 (paratipo). 